TRANSACTIONS OF THE

AMERICAN MATHEMATICAL SOCIETY

Volume 360, Number 9, September 2008, Pages 4541-4568

S 0002-9947(08)04478-4

Article electronically published on April 4, 2008

\title{
HOW OFTEN ARE TWO PERMUTATIONS COMPARABLE?
}

\author{
ADAM HAMMETT AND BORIS PITTEL
}

\begin{abstract}
Two permutations of $[n]$ are comparable in the Bruhat order if one is closer, in a natural way, to the identity permutation, $12 \cdots n$, than the other. We show that the number of comparable pairs is of order $(n !)^{2} / n^{2}$ at most, and $(n !)^{2}(0.708)^{n}$ at least. For the related weak order, the corresponding bounds are $(n !)^{2}(0.362)^{n}$ and $(n !)^{2} \prod_{i=1}^{n}(H(i) / i)$, where $H(i):=\sum_{j=1}^{i} 1 / j$. In light of numerical experiments, we conjecture that for each order the upper bound is qualitatively close to the actual number of comparable pairs.
\end{abstract}

\section{INTRODUCTION}

Let $n \geq 1$ be an integer. Two permutations of $[n]:=\{1, \ldots, n\}$ are comparable in the Bruhat order if one can be obtained from the other by a sequence of transpositions of pairs of elements forming an inversion. Here is a precise definition of the Bruhat order on the set of permutations $S_{n}$ (see Stanley [24, p. 172, ex. 75 . a], Humphreys [15, p. 119]). If $\omega=\omega(1) \cdots \omega(n) \in S_{n}$, then a reduction of $\omega$ is a permutation obtained from $\omega$ by interchanging some $\omega(i)$ with some $\omega(j)$ provided $i<j$ and $\omega(i)>\omega(j)$. We say that $\pi \leq \sigma$ in the Bruhat order if there is a chain $\sigma=\omega_{1} \rightarrow \omega_{2} \rightarrow \cdots \rightarrow \omega_{s}=\pi$, where each $\omega_{t}$ is a reduction of $\omega_{t-1}$. The number of inversions in $\omega_{t}$ strictly decreases with $t$. Indeed, one can show that if $\omega_{2}$ is a reduction of $\omega_{1}$ via the interchange $\omega_{1}(i) \leftrightarrow \omega_{1}(j), i<j$, then

$$
\begin{gathered}
\operatorname{inv}\left(\omega_{1}\right)=\operatorname{inv}\left(\omega_{2}\right)+2 N\left(\omega_{1}\right)+1 \\
N\left(\omega_{1}\right):=\left|\left\{k: i<k<j, \omega_{1}(i)>\omega_{1}(k)>\omega_{1}(j)\right\}\right| ;
\end{gathered}
$$

here inv $\left(\omega_{1}\right)$, say, is the number of inversions in $\omega_{1}$ (see Björner and Brenti [3]). Figure 1 illustrates this poset on $S_{3}$ and $S_{4}$. The Bruhat order notion can be extended to other Coxeter groups (see Björner [6], Deodhar [10], and [3, p. 63] for historical background), but we will be dealing with the symmetric group $S_{n}$ only.

There exist efficient algorithms for checking Bruhat comparability, which can all be traced back to an algorithmic comparability criterion due to Ehresmann (1934) [12] (see also Knuth [18, Björner and Brenti [3]). The Ehresmann "tableau criterion" states that $\pi \leq \sigma$ if and only if $\pi_{i, j} \leq \sigma_{i, j}$ for all $1 \leq i \leq j \leq n-1$, where $\pi_{i, j}$ and $\sigma_{i, j}$ are the $i$-th entry in the increasing rearrangement of $\pi(1), \ldots, \pi(j)$ and of $\sigma(1), \ldots, \sigma(j)$. These arrangements form two staircase tableaux, hence the

Received by the editors January 31, 2006.

2000 Mathematics Subject Classification. Primary 05A05, 05A16, 06A07, 60C05; Secondary $20 \mathrm{~B} 99$.

Key words and phrases. Bruhat ordering, weak ordering, permutations, partially ordered sets.

The first author was supported in part by NSF grant DMS-0104104.

The second author was supported in part by NSF grants DMS-0104104 and DMS-0406024.

(C) 2008 American Mathematical Society 

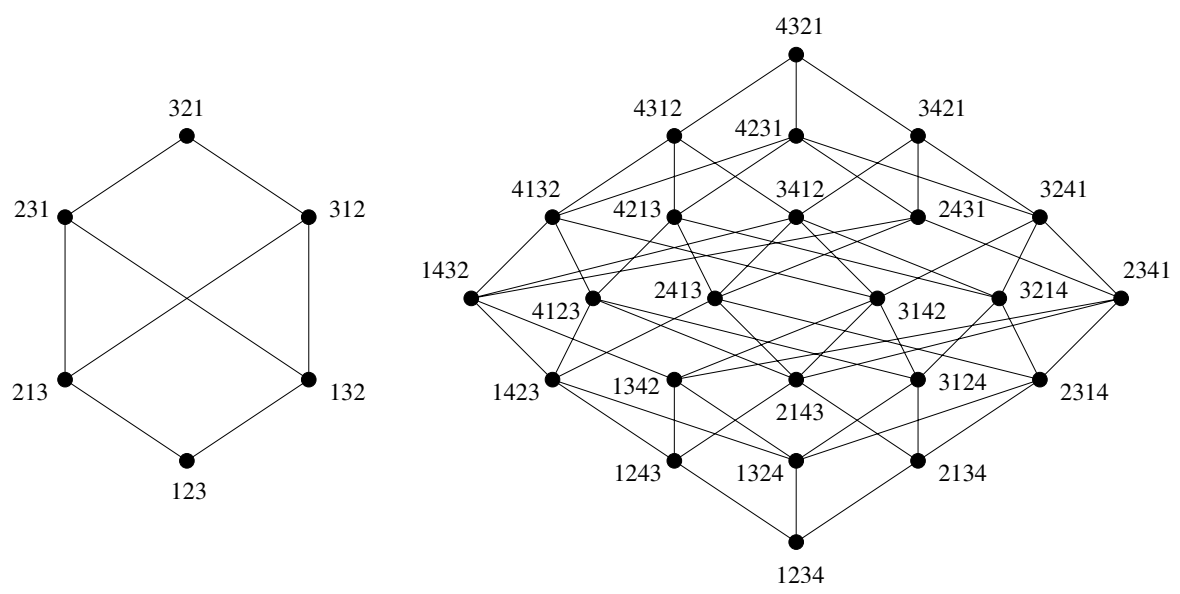

Figure 1. The Bruhat order on $S_{3}$ and $S_{4}$

term "tableau criterion". For example, $41523>21534$ is verified by elementwise comparisons of the two tableaux

$\begin{array}{llll}1 & 2 & 4 & 5 \\ 1 & 4 & 5 & \\ 1 & 4 & & \\ 4 & & & \end{array}$

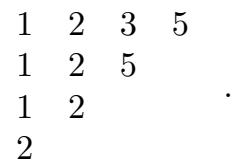

Also, it is well known that Ehresmann's criterion is equivalent to the $(0,1)$-matrix criterion. It involves comparing the number of 1 's contained in certain submatrices of the (0,1)-permutation matrices representing $\pi$ and $\sigma$ (see Bóna [8, 3]). Later, Björner and Brenti [4] were able to improve on the result of [12, giving a tableau criterion that requires fewer operations. Very recently, Drake, Gerrish and Skandera 11] have found two new comparability criteria, involving totally nonnegative polynomials and the Schur functions respectively. We are aware of other criteria (see [6], Fulton [14, pp. 173-177], Lascoux and Schützenberger [19, [10]), but we found the $(0,1)$-matrix and Ehresmann criteria most amenable to probabilistic study.

The $(0,1)$-matrix criterion for Bruhat order on $S_{n}$ says that for $\pi, \sigma \in S_{n}, \pi \leq \sigma$ if and only if for all $i, j \leq n$, the number of $\pi(1), \ldots, \pi(i)$ that are at most $j$ exceeds (or equals) the number of $\sigma(1), \ldots, \sigma(i)$ that are at most $j$ (see [8] for this version). It is referred to as the $(0,1)$-matrix criterion because of the following recasting of this condition: let $M(\pi), M(\sigma)$ be the permutation matrices corresponding to $\pi$, $\sigma$, so that for instance the $(i, j)$-entry of $M(\pi)$ is 1 if $\pi(j)=i$ and 0 otherwise. Here, we are labeling columns $1,2, \ldots, n$ when reading from left to right, and rows are labeled $1,2, \ldots, n$ when reading from bottom to top so that this interpretation is like placing ones at points $(i, \pi(i))$ of the $n \times n$ integer lattice and zeroes elsewhere. Denoting submatrices of $M(\cdot)$ corresponding to rows $I$ and columns $J$ by $M(\cdot)_{I, J}$, this criterion says that $\pi \leq \sigma$ if and only if for all $i, j \leq n$, the number of ones in $M(\pi)_{[i],[j]}$ is at least the number of ones in $M(\sigma)_{[i],[j]}$ (see [11] for this version).

An effective way of visualizing this criterion is to imagine the matrices $M(\pi)$ and $M(\sigma)$ as being superimposed on one another into a single matrix, $M(\pi, \sigma)$, with the ones for $M(\pi)$ represented by $\times$ 's ("crosses"), the ones for $M(\sigma)$ by o's ("balls") 


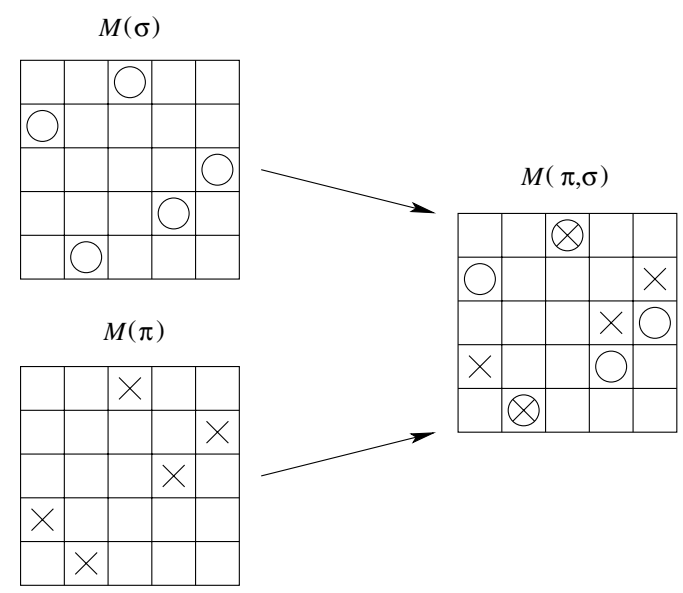

FiguRE 2. Superimposing $M(\pi)$ and $M(\sigma)$ to form $M(\pi, \sigma)$

and the zeroes for both by empty entries. Note that some entries of $M(\pi, \sigma)$ may be occupied by both a cross and a ball. Then the $(0,1)$-matrix criterion says that $\pi \leq \sigma$ if and only if every southwest submatrix of $M(\pi, \sigma)$ contains at least as many crosses as balls. Here, in the notation above, a southwest submatrix is a submatrix $M(\pi, \sigma)_{[i],[j]}$ of $M(\pi, \sigma)$ for some $i, j \leq n$. It is clear that we could also check $\pi \leq \sigma$ by checking that crosses are at least as numerous as balls in every northeast submatrix of $M(\pi, \sigma)$. Likewise, $\pi \leq \sigma$ if and only if balls are at least as numerous as crosses in every northwest submatrix of $M(\pi, \sigma)$, or similarly balls are at least as numerous as crosses in every southeast submatrix of $M(\pi, \sigma)$. Parts of all four of these equivalent conditions will be used in our proofs. As a quick example, with $\pi=21534$ and $\sigma=41523, \pi<\sigma$ is checked by examining southwest submatrices of $M(\pi, \sigma)$ in Figure 2. Also, the superimposing of $M(\pi)$ with $M(\sigma)$ to form $M(\pi, \sigma)$ is illustrated in this figure.

In this note, we use the $(0,1)$-matrix and the Ehresmann criteria to obtain upper and lower bounds for the number of pairs $(\pi, \sigma)$ with $\pi \leq \sigma$.

Theorem 1.1. Let $n \geq 1$ be an integer, and let $\pi, \sigma \in S_{n}$ be selected independently and uniformly at random. Then there exist universal constants $c_{1}, c_{2}>0$ such that

$$
c_{1}(0.708)^{n} \leq P(\pi \leq \sigma) \leq c_{2} / n^{2} .
$$

Equivalently, the number of pairs $(\pi, \sigma)$ with $\pi \leq \sigma$ is sandwiched between the counts $c_{1}(0.708)^{n}(n !)^{2}$ and $c_{2} n^{-2}(n !)^{2}$. The lower bound follows from a sufficient condition derived from the $(0,1)$-matrix criterion, and a computer-aided tabulation of an attendant function of a smallish integer argument. Empirical estimates based on generating pairs of random permutations suggest that $P(\pi \leq \sigma)$ is of order $n^{-(2+\delta)}$, for $\delta$ close to 0.5 from above. So apparently it is the upper bound which comes close to the true proportion $P(\pi \leq \sigma)$. It is certain that the constant 0.708 can be further improved, but we do not know if our method could be extended to deliver a lower bound $(1-o(1))^{n}$. A lower bound $n^{-a}$, a qualitative match of the upper bound, seems out of sight presently.

Then we turn to the modified order on $S_{n}$, the weak order " $\preceq$ ". Here $\pi \preceq \sigma$ if there is a chain $\sigma=\omega_{1} \rightarrow \omega_{2} \rightarrow \cdots \rightarrow \omega_{s}=\pi$, where each $\omega_{t}$ is a simple reduction 


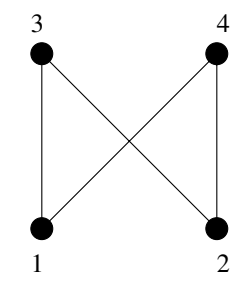

FIGURE 3 . The permutation-induced poset $\mathcal{P}(2143)$

of $\omega_{t-1}$, i.e. obtained from $\omega_{t-1}$ by transposing two adjacent elements $\omega_{t-1}(i)$, $\omega_{t-1}(i+1)$ with $\omega_{t-1}(i)>\omega_{t-1}(i+1)$. Since at each step the number of inversions decreases by 1 , all chains connecting $\sigma$ and $\pi$ have the same length.

Theorem 1.2. Let $P_{n}^{*}:=P(\pi \preceq \sigma)$. Then $P_{n}^{*}$ is submultiplicative, i.e. $P_{n_{1}+n_{2}}^{*} \leq$ $P_{n_{1}}^{*} P_{n_{2}}^{*}$. Consequently, there exists $\rho=\lim \sqrt[n]{P_{n}^{*}}$. Furthermore, there exists an absolute constant $c>0$ such that

$$
\prod_{i=1}^{n}(H(i) / i) \leq P_{n}^{*} \leq c(0.362)^{n},
$$

where $H(i):=\sum_{j=1}^{i} 1 / j$. Consequently, $\rho \leq 0.362$.

The proof of the upper bound is parallel to that of Theorem 1.1, lower bound, while the lower bound follows from an inversion set criterion inspired by discussion of the weak order by Berge 11. Empirical estimates indicate that $\rho$ is close to 0.3 . So here too the upper bound seems to be qualitatively close to the actual probability $P_{n}^{*}$, and our lower bound, though superior to the trivial bound $1 / n$ !, is decreasing superexponentially fast with $n$, which makes us believe that there ought to be a way to vastly improve it.

Paradoxically, it is the lower bound that required a deeper combinatorial insight. Clearly the number of $\pi$ 's below (or equal to) $\sigma$ equals $e(\mathcal{P})$, the total number of linear extensions of $\mathcal{P}=\mathcal{P}(\sigma)$, the poset induced by $\sigma$. (The important notion of $\mathcal{P}(\sigma)$ was brought to our attention by Sergey Fomin [13].) We prove that for any poset $\mathcal{P}$ of cardinality $n$,

$$
e(\mathcal{P}) \geq n ! / \prod_{i \in \mathcal{P}} d(i),
$$

where $d(i):=\mid\{j \in \mathcal{P}: j \leq i$ in $\mathcal{P}\} \mid$. (This bound is an exact value of $e(\mathcal{P})$ if the Hasse diagram is a (directed) rooted tree, Knuth [18, sect. 5.1.4, ex. 20], or a forest of such trees, Björner and Wachs [5.) The bound (1.1) for $e(\mathcal{P}(\sigma))$ together with the independence of sequential ranks in the uniform permutation were the key ingredients in the proof of Theorem 1.2, lower bound.

Miklós Bóna [7 has informed us that this general lower bound for $e(\mathcal{P})$ had been stated by Richard Stanley as a level 2 exercise in [24, p. 312, ex. 1] without a solution. We have decided to keep the proof in the paper, since we could not find a published proof anywhere either. The classic hook formula provides an example of a poset $\mathcal{P}$ for which (1.1) is markedly below $e(\mathcal{P})$. It remains to be seen whether (1.1) can be strengthened in general, or at least for $\mathcal{P}(\sigma)$. As an illustration, $\mathcal{P}=\mathcal{P}(2143)$ has the Hasse diagram appearing in Figure 3, Then $e(\mathcal{P})=4$, but (1.1) delivers 
only

$$
e(\mathcal{P}) \geq 24 / 9, \Longrightarrow e(\mathcal{P}) \geq 3 .
$$

In conclusion we mention [21] and 22, where the "probability-of-comparability" problems were solved for the poset of integer partitions of $n$ under the dominance order, and for the poset of set partitions of $[n]$ ordered by refinement.

\section{Proof of Theorem 1.1, upper bound}

We need to show that

$$
P(\pi \leq \sigma)=O\left(n^{-2}\right) .
$$

The argument is based on the $(0,1)$-matrix criterion. We assume that $n$ is even. Only minor modifications are necessary for $n$ odd.

Step 1. The $(0,1)$-matrix criterion requires that a set of $n^{2}$ conditions are met. The challenge is to select a subset of those conditions which meets two conflicting demands. It has to be sufficiently simple so that we can compute (estimate) the probability that the random pair $(\pi, \sigma)$ satisfies all the chosen conditions. On the other hand, collectively these conditions need to be quite stringent for this probability to be $o(1)$. In our first advance we were able (via Ehresmann's criterion) to get a bound $O\left(n^{-1 / 2}\right)$ by using about $2 n^{1 / 2}$ conditions. We are about to describe a set of $2 n$ conditions that does the job.

Let us split the matrices $M(\pi, \sigma), M(\pi)$ and $M(\sigma)$ into 4 submatrices of equal size $n / 2 \times n / 2$ - the southwest, northeast, northwest and southeast corners, denoting them $M_{s w}(\cdot), M_{n e}(\cdot), M_{n w}(\cdot)$ and $M_{s e}(\cdot)$, respectively. In the southwest corner $M_{s w}(\pi, \sigma)$, we restrict our attention to southwest submatrices of the form $i \times n / 2$, $i=1, \ldots, n / 2$. If $\pi \leq \sigma$, then as we read off rows of $M_{s w}(\pi, \sigma)$ from bottom to top, keeping track of the total number of balls and crosses encountered thus far, at any intermediate point we must have at least as many crosses as balls. Let us denote the set of pairs $(\pi, \sigma)$ such that this occurs by $\mathcal{E}_{s w}$. We draw analogous conclusions for the northeast corner, reading rows from top to bottom, and we denote by $\mathcal{E}_{n e}$ the set of pairs $(\pi, \sigma)$ satisfying this condition.

Similarly, we can read columns from left to right in the northwest corner, and here we must always have at least as many balls as crosses. Denote the set of these pairs $(\pi, \sigma)$ by $\mathcal{E}_{n w}$. The same condition holds for the southeast corner when we read columns from right to left. Denote the set of these pairs $(\pi, \sigma)$ by $\mathcal{E}_{s e}$. Letting $\mathcal{E}$ denote the set of pairs $(\pi, \sigma)$ satisfying all four of the conditions above, we get

$$
\{\pi \leq \sigma\} \subseteq \mathcal{E}=\mathcal{E}_{s w} \cap \mathcal{E}_{n e} \cap \mathcal{E}_{n w} \cap \mathcal{E}_{s e} .
$$

Pairs of permutations in $\mathcal{E}$ satisfy $2 n$ of the $n^{2}$ conditions required by the $(0,1)$ matrix criterion. Unlike the set $\{\pi \leq \sigma\}$, we are able to compute $|\mathcal{E}|$, and to show that $P(\mathcal{E})=(n !)^{-2}|\mathcal{E}|=O\left(n^{-2}\right)$. Figure 4 is a graphical visualization of the reading-off process that generates the restrictions defining the set $\mathcal{E}$.

If a row (column) of a submatrix $M(\pi)_{I, J}\left(M(\sigma)_{I, J}\right.$ resp.) contains a marked entry, we say that it supports the submatrix. Clearly the number of supporting rows (columns) equals the number of marked entries in $M(\pi)_{I, J}\left(M(\sigma)_{I, J}\right.$ resp.). Now, given $\pi, \sigma$, let $M_{1}=M_{1}(\pi), M_{2}=M_{2}(\sigma)$ denote the total number of rows that support $M_{s w}(\pi)$ and $M_{s w}(\sigma)$, respectively. Then $M_{n w}(\pi), M_{n w}(\sigma)$ are supported by $M_{3}=n / 2-M_{1}$ columns and by $M_{4}=n / 2-M_{2}$ columns, respectively. The same 


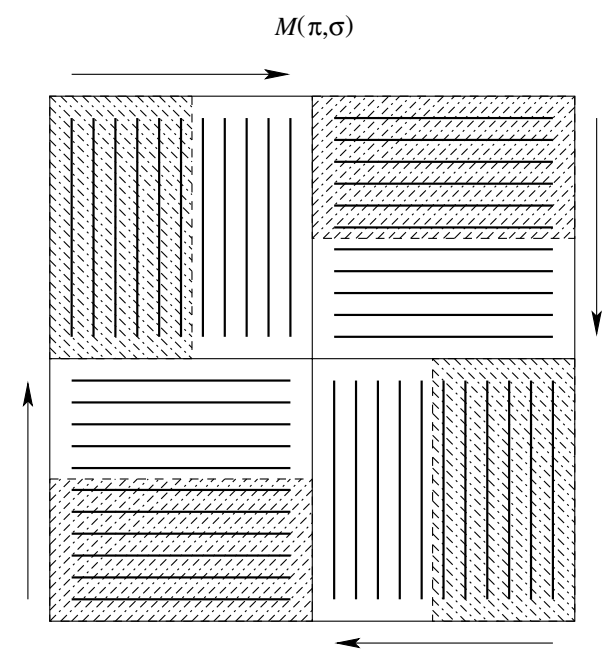

FIGURE 4. Finding a necessary condition for $\pi \leq \sigma$

holds for the southeast corners of $M(\pi)$ and $M(\sigma)$. Obviously the northeast submatrices of $M(\pi)$ and $M(\sigma)$ are supported by $M_{1}$ rows and $M_{2}$ rows, respectively. Then we have

$$
\begin{gathered}
P(\mathcal{E})=\sum_{m_{1}, m_{2}} P\left(\mathcal{E} \cap \mathcal{A}\left(m_{1}, m_{2}\right)\right), \\
\mathcal{A}\left(m_{1}, m_{2}\right):=\left\{(\pi, \sigma): M_{1}=m_{1}, M_{2}=m_{2}\right\} .
\end{gathered}
$$

Clearly $\mathcal{E} \cap \mathcal{A}\left(m_{1}, m_{2}\right)=\emptyset$ if $m_{1}<m_{2}$. We claim that, for $m_{1} \geq m_{2}$,

$$
P\left(\mathcal{E} \cap \mathcal{A}\left(m_{1}, m_{2}\right)\right)=\left[\frac{\left(m_{1}-m_{2}+1\right)(n / 2+1)}{\left(m_{3}+1\right)\left(m_{1}+1\right)}\right]^{4} \cdot \frac{\prod_{i=1}^{4}\left(\begin{array}{c}
n / 2 \\
m_{i}
\end{array}\right)}{\left(\begin{array}{c}
n \\
n / 2
\end{array}\right)^{2}} .
$$

Here and below, $m_{3}:=n / 2-m_{1}$ and $m_{4}:=n / 2-m_{2}$ stand for generic values of $M_{3}$ and $M_{4}$ in the event $\mathcal{A}\left(m_{1}, m_{2}\right)$.

To prove ( $\dagger$ ), let us count the number of pairs $(\pi, \sigma)$ in $\mathcal{E} \cap \mathcal{A}\left(m_{1}, m_{2}\right)$. First consider the southwest corner, $M_{s w}(\pi, \sigma)$. Introduce $L_{1}=L_{1}(\pi, \sigma)$, the number of rows supporting both $M_{s w}(\pi)$ and $M_{s w}(\sigma)$. So $L_{1}$ is the number of rows in the southwest corner $M_{s w}(\pi, \sigma)$ containing both a cross and a ball. Suppose that we are on the event $\left\{L_{1}=\ell_{1}\right\}$. We choose $\ell_{1}$ rows to support both $M_{s w}(\pi)$ and $M_{s w}(\sigma)$ from the $n / 2$ first rows. Then, we choose $\left(m_{1}-\ell_{1}+m_{2}-\ell_{1}\right)$ more rows from the remaining $\left(n / 2-\ell_{1}\right)$ rows. Each of these secondary rows is to support either $M_{s w}(\pi)$ or $M_{s w}(\sigma)$, but not both. This step can be done in

$$
\left(\begin{array}{c}
n / 2 \\
\ell_{1}
\end{array}\right)\left(\begin{array}{c}
n / 2-\ell_{1} \\
m_{1}-\ell_{1}+m_{2}-\ell_{1}
\end{array}\right)
$$

ways. Next, we partition the set of $\left(m_{1}-\ell_{1}+m_{2}-\ell_{1}\right)$ secondary rows into two row subsets of cardinality $\left(m_{1}-\ell_{1}\right)$ (rows to contain crosses) and $\left(m_{2}-\ell_{1}\right)$ (rows to contain balls) that will support $M_{s w}(\pi)$ and $M_{s w}(\sigma)$, accompanying the $\ell_{1}$ primary rows supporting both submatrices. We can visualize each of the resulting row selections as a subsequence of $(1, \ldots, n / 2)$ which is a disjoint union of two subsequences, one with $\ell_{1}$ elements labeled by a ball and a cross, and another with 
$\left(m_{1}-\ell_{1}+m_{2}-\ell_{1}\right)$ elements, $\left(m_{1}-\ell_{1}\right)$ labeled by crosses and the remaining $\left(m_{2}-\ell_{1}\right)$ elements labeled by balls. The condition $\mathcal{E}_{s w}$ is equivalent to the restriction: moving along the subsequence from left to right, at each point the number of crosses is not to fall below the number of balls. Obviously, no double-marked element can cause a violation of this condition. Thus, our task is reduced to the determination of the number of $\left(m_{1}-\ell_{1}+m_{2}-\ell_{1}\right)$-long sequences of $m_{1}-\ell_{1}$ crosses and $m_{2}-\ell_{1}$ balls such that at no point is the number of crosses strictly less than the number of balls. By the classic ballot theorem (see Takacs [25, pp. 2-7]), the total number of such sequences equals

$$
\begin{aligned}
\frac{\left(m_{1}-\ell_{1}+1\right)-\left(m_{2}-\ell_{1}\right)}{\left(m_{1}-\ell_{1}+1\right)+\left(m_{2}-\ell_{1}\right)}\left(\begin{array}{c}
m_{1}-\ell_{1}+m_{2}-\ell_{1}+1 \\
m_{1}-\ell_{1}+1
\end{array}\right) & \\
= & \frac{m_{1}-m_{2}+1}{m_{1}-\ell_{1}+1}\left(\begin{array}{c}
m_{1}-\ell_{1}+m_{2}-\ell_{1} \\
m_{1}-\ell_{1}
\end{array}\right) .
\end{aligned}
$$

The second binomial coefficient is the total number of $\left(m_{1}-\ell_{1}+m_{2}-\ell_{1}\right)$-long sequences of $\left(m_{1}-\ell_{1}\right)$ crosses and $\left(m_{2}-\ell_{1}\right)$ balls. So the second fraction is the probability that the sequence chosen uniformly at random among all such sequences meets the ballot theorem condition. The total number of ways to designate the rows supporting $M_{s w}(\pi)$ and $M_{s w}(\sigma)$, subject to the condition $\mathcal{E}_{s w}$, is the product of two counts, namely

$$
\begin{aligned}
\left(\begin{array}{c}
n / 2 \\
\ell_{1}
\end{array}\right)\left(\begin{array}{c}
n / 2-\ell_{1} \\
m_{1}-\ell_{1}+m_{2}-\ell_{1}
\end{array}\right) & \left(\begin{array}{c}
m_{1}-\ell_{1}+m_{2}-\ell_{1} \\
m_{1}-\ell_{1}
\end{array}\right) \frac{m_{1}-m_{2}+1}{m_{1}-\ell_{1}+1} \\
= & \frac{m_{1}-m_{2}+1}{n / 2-m_{2}+1}\left(\begin{array}{c}
n / 2 \\
m_{2}
\end{array}\right)\left(\begin{array}{c}
m_{2} \\
\ell_{1}
\end{array}\right)\left(\begin{array}{c}
n / 2-m_{2}+1 \\
m_{1}-\ell_{1}+1
\end{array}\right) .
\end{aligned}
$$

Summing this last expression over all $\ell_{1} \leq m_{2}$, we obtain

$$
\begin{aligned}
\frac{m_{1}-m_{2}+1}{n / 2-m_{2}+1}\left(\begin{array}{c}
n / 2 \\
m_{2}
\end{array}\right) \sum_{\ell_{1} \leq m_{2}}\left(\begin{array}{c}
m_{2} \\
\ell_{1}
\end{array}\right)\left(\begin{array}{c}
n / 2-m_{2}+1 \\
m_{1}-\ell_{1}+1
\end{array}\right) \\
=\frac{m_{1}-m_{2}+1}{n / 2-m_{2}+1}\left(\begin{array}{c}
n / 2 \\
m_{2}
\end{array}\right)\left(\begin{array}{c}
n / 2+1 \\
m_{1}+1
\end{array}\right) \\
=\frac{\left(m_{1}-m_{2}+1\right)(n / 2+1)}{\left(n / 2-m_{2}+1\right)\left(m_{1}+1\right)}\left(\begin{array}{c}
n / 2 \\
m_{1}
\end{array}\right)\left(\begin{array}{c}
n / 2 \\
m_{2}
\end{array}\right) .
\end{aligned}
$$

Here, in the first equality, we have used the binomial theorem. The product of the two binomial coefficients in the final count (2.2) is the total number of row selections from the first $n / 2$ rows, $m_{1}$ to contain crosses and $m_{2}$ to contain balls. So the fraction preceding these two binomial factors is the probability that a particular row selection chosen uniformly at random from all such row selections satisfies our ballot condition "crosses never fall below balls". Equivalently, by the very derivation, the expression (2.2) is the total number of paths $(X(t), Y(t))_{0 \leq t \leq n / 2}$ on the square lattice connecting $(0,0)$ and $\left(m_{1}, m_{2}\right)$ such that $X(t+1)-X(t), Y(t+1)-Y(t) \in$ $\{0,1\}$, and $X(t) \geq Y(t)$ for every $t$. (To be sure, if $X(t+1)-X(t)=1$ and $Y(t+1)-Y(t)=1$, the corresponding move is a combination of horizontal and vertical unit moves.)

Likewise, we consider the northeast corner, $M_{n e}(\pi, \sigma)$. We introduce $L_{2}=$ $L_{2}(\pi, \sigma)$, the number of rows in $M_{n e}(\pi, \sigma)$ containing both a cross and a ball. By initially restricting to the event $\left\{L_{2}=\ell_{2}\right\}$, then later summing over all $\ell_{2} \leq m_{2}$, we obtain another factor (2.2). Analogously, a third and fourth factor (2.2) comes 


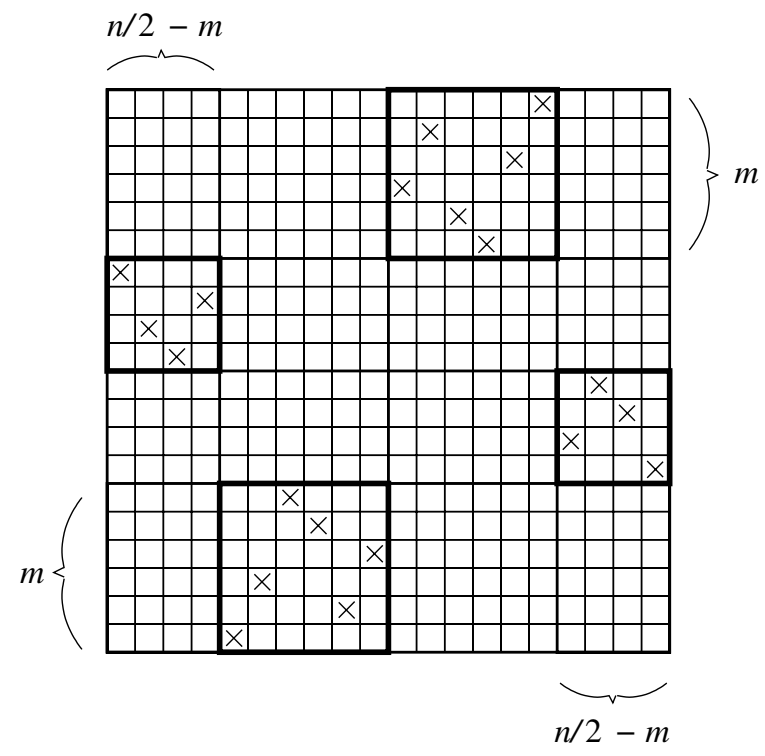

FiguRE 5. Selection of first $m=m_{1}(n / 2-m$ resp.) rows (columns resp.) in corners to support $M(\pi)$

from considering columns in the northwest and southeast corners, $M_{n w}(\pi, \sigma)$ and $M_{s e}(\pi, \sigma)$. Importantly, the row selections for the southwest and the northeast submatrices do not interfere with the column selections for the northwest and the southeast corners. So by multiplying these four factors (2.2) we obtain the total number of row and column selections on the event $\mathcal{A}\left(m_{1}, m_{2}\right)$ subject to all four restrictions defining $\mathcal{E}$ !

Once such a row-column selection has been made, we have determined which rows and columns support the four submatrices of $M(\pi)$ and $M(\sigma)$. Consider, for instance, the southwest corner of $M(\pi)$. We have selected $m_{1}$ rows (from the first $n / 2$ rows) supporting $M_{s w}(\pi)$, and we have selected $m_{3}$ columns (from the first $n / 2$ columns) supporting $M_{n w}(\pi)$. Then it is the remaining $n / 2-m_{3}=m_{1}$ columns that support $M_{s w}(\pi)$. The number of ways to match these $m_{1}$ rows and $m_{1}$ columns, thus to determine $M_{s w}(\pi)$ completely, is $m_{1}$ !. The northeast corner contributes another $m_{1}$ !, while each of the two other corners contributes $m_{3}$ !, whence the overall matching factor is $\left(m_{1} ! m_{3} !\right)^{2}$. The matching factor for $\sigma$ is $\left(m_{2} ! m_{4} !\right)^{2}$. Multiplying the number of admissible row-column selections by the resulting $\prod_{i=1}^{4}\left(m_{i} !\right)^{2}$ and dividing by $(n !)^{2}$, we obtain

$$
P\left(\mathcal{E} \cap \mathcal{A}\left(m_{1}, m_{2}\right)\right)=\left[\frac{\left(m_{1}-m_{2}+1\right)(n / 2+1)}{\left(n / 2-m_{2}+1\right)\left(m_{1}+1\right)}\left(\begin{array}{c}
n / 2 \\
m_{1}
\end{array}\right)\left(\begin{array}{c}
n / 2 \\
m_{2}
\end{array}\right)\right]^{4} \cdot \frac{\prod_{i=1}^{4}\left(m_{i} !\right)^{2}}{(n !)^{2}}
$$

which is equivalent to $(\dagger)$. Figure 5 is a graphical explanation of this matching factor. In it, we show the matrix $M(\pi)$ in a case when in the southwest and the northeast squares $\pi$ is supported by the bottom $m\left(=m_{1}\right)$ and the top $m$ rows, respectively; likewise, in the northwest and the southeast squares $\pi$ is supported by the $n / 2-m$ leftmost and the $n / 2-m$ rightmost columns, respectively. 
Step 2. Let us show that (2.1) and (†) imply

$$
P(\mathcal{E}) \leq E\left[\frac{\left(M_{1}-M_{2}+1\right)^{4}(n / 2+1)^{4}}{\left(n / 2-M_{2}+1\right)^{4}\left(M_{1}+1\right)^{4}}\right] .
$$

First, $M_{1}$ and $M_{2}$ are independent with

$$
P\left(M_{i}=m_{i}\right)=\frac{\left(\begin{array}{c}
n / 2 \\
m_{i}
\end{array}\right)^{2}}{\left(\begin{array}{c}
n \\
n / 2
\end{array}\right)}, \quad i=1,2 .
$$

Indeed, $M_{i}$ obviously equals the cardinality of the intersection with $[n / 2]$ of a uniformly random subset of size $n / 2$ from $[n]$, which directly implies these formulas. Thus, each $M_{i}$ has the hypergeometric distribution with parameters $n / 2, n / 2, n / 2$; in other words, $M_{i}$ has the same distribution as the number of red balls in a uniformly random sample of $n / 2$ balls from an urn containing $n / 2$ red balls and $n / 2$ white balls. By the independence of $M_{1}$ and $M_{2}$, we obtain

$$
P\left(M_{1}=m_{1}, M_{2}=m_{2}\right)=\frac{\left(\begin{array}{c}
n / 2 \\
m_{1}
\end{array}\right)^{2}\left(\begin{array}{c}
n / 2 \\
m_{2}
\end{array}\right)^{2}}{\left(\begin{array}{c}
n \\
n / 2
\end{array}\right)^{2}} .
$$

It remains to observe that (2.1) and ( $\dagger$ ) imply

$$
\begin{aligned}
P(\mathcal{E}) & =\sum_{m_{1} \geq m_{2}} \frac{\left(m_{1}-m_{2}+1\right)^{4}(n / 2+1)^{4}}{\left(n / 2-m_{2}+1\right)^{4}\left(m_{1}+1\right)^{4}} \cdot P\left(M_{1}=m_{1}, M_{2}=m_{2}\right) \\
& \leq \sum_{m_{1}, m_{2}} \frac{\left(m_{1}-m_{2}+1\right)^{4}(n / 2+1)^{4}}{\left(n / 2-m_{2}+1\right)^{4}\left(m_{1}+1\right)^{4}} \cdot P\left(M_{1}=m_{1}, M_{2}=m_{2}\right) \\
& =E\left[\frac{\left(M_{1}-M_{2}+1\right)^{4}(n / 2+1)^{4}}{\left(n / 2-M_{2}+1\right)^{4}\left(M_{1}+1\right)^{4}}\right],
\end{aligned}
$$

and $(\ddagger)$ is proved.

Step 3. The advantage of $(\ddagger)$ is that it allows us to use probabilistic tools exploiting the independence of the random variables $M_{1}$ and $M_{2}$. Typically the $M_{i}$ 's are close to $n / 4$, while $\left|M_{1}-M_{2}\right|$ is of order $n^{1 / 2}$ at most. So, in view of $(\ddagger)$ we expect that $P(\mathcal{E})=O\left(n^{-2}\right)$.

We now make this argument rigorous. First of all, by the "sample-from-urn" interpretation of $M_{i}$,

$$
E\left[M_{i}\right]=\frac{n}{2} \frac{\left(\begin{array}{c}
n-1 \\
n / 2-1
\end{array}\right)}{\left(\begin{array}{c}
n \\
n / 2
\end{array}\right)}=n / 4 .
$$

Then (see Janson et al. [16, p. 29]) the probability generating function of $M_{i}$ is dominated by that of $\operatorname{Bin}(n, 1 / 4)$, and consequently for each $t \geq 0$ we have

$$
P\left(\left|M_{i}-n / 4\right| \geq t\right)=O\left(\exp \left(-4 t^{2} / n\right)\right) .
$$

Hence, setting $t=n^{2 / 3}$ we see that

$$
P\left(n / 4-n^{2 / 3}<M_{i}<n / 4+n^{2 / 3}\right) \geq 1-e^{-c n^{1 / 3}},
$$


for some absolute constant $c>0$. Introduce the event

$$
A_{n}=\bigcap_{i=1}^{2}\left\{n / 4-n^{2 / 3}<M_{i}<n / 4+n^{2 / 3}\right\} \text {. }
$$

Combining the estimates for $M_{i}$, we see that for some absolute constant $c_{1}>0$,

$$
P\left(A_{n}\right) \geq 1-e^{-c_{1} n^{1 / 3}} .
$$

Now the random variable in $(\ddagger)$, call it $X_{n}$, is bounded by 1 , and on the event $A_{n}$, within a factor of $1+O\left(n^{-1 / 3}\right)$,

$$
X_{n}=\left(\frac{4}{n}\right)^{8}\left(M_{1}-M_{2}+1\right)^{4}(n / 2+1)^{4} \text {. }
$$

Therefore

$$
P(\mathcal{E}) \leq\left(\frac{5}{n}\right)^{8}(n / 2+1)^{4} E\left[\left(M_{1}-M_{2}+1\right)^{4}\right]+O\left(e^{-c_{1} n^{1 / 3}}\right) .
$$

It remains to prove that this expected value is $O\left(n^{2}\right)$. Introduce $\bar{M}_{i}=M_{i}-E\left[M_{i}\right]$, $i=1,2$. Then

$$
\left(M_{1}-M_{2}+1\right)^{4}=\left(\bar{M}_{1}-\bar{M}_{2}+1\right)^{4} \leq 27\left(\bar{M}_{1}^{4}+\bar{M}_{2}^{4}+1\right),
$$

as

$$
(a+b+c)^{2} \leq 3\left(a^{2}+b^{2}+c^{2}\right)
$$

We now demonstrate that $E\left[\bar{M}_{i}^{4}\right]=O\left(n^{2}\right)$. To this end, notice first that $E\left[\bar{M}_{i}^{2}\right]$ is of order $n$ exactly. Indeed, extending the computation in (2.3),

$$
\begin{aligned}
E\left[M_{i}\left(M_{i}-1\right)\right] & =\frac{n}{2}\left(\frac{n}{2}-1\right) \frac{\left(\begin{array}{c}
n-2 \\
n / 2-2
\end{array}\right)}{\left(\begin{array}{c}
n \\
n / 2
\end{array}\right)} \\
& =\frac{n(n-2)^{2}}{16(n-1)} .
\end{aligned}
$$

Therefore

$$
\begin{aligned}
E\left[\bar{M}_{i}^{2}\right] & =\operatorname{Var}\left[M_{i}\right] \\
& =E\left[M_{i}\left(M_{i}-1\right)\right]+E\left[M_{i}\right]-E^{2}\left[M_{i}\right] \\
& =\frac{n(n-2)^{2}}{16(n-1)}+\frac{n}{4}-\frac{n^{2}}{16} \\
& =\frac{n}{16}+O(1) .
\end{aligned}
$$

Furthermore, as a special instance of the hypergeometrically distributed random variable, $M_{i}$ has the same distribution as the sum of $n / 2$ independent Bernoulli variables $Y_{j} \in\{0,1\}$ (see Vatutin and Mikhailov [20, alternatively [16, p. 30]). Therefore, (2.4) and the Lindeberg-Feller Central Limit Theorem imply

$$
\frac{\bar{M}_{i}}{\sqrt{n / 16}} \Longrightarrow \mathcal{N}(0,1)
$$

where $\mathcal{N}(0,1)$ is the standard normal random variable. In fact, since

$$
\frac{Y_{j}-E\left[Y_{j}\right]}{\sqrt{n / 16}} \rightarrow 0, \quad n \rightarrow \infty
$$




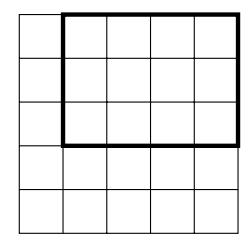

Figure $6 . G_{5}$ and an emboldened subgrid $C$

we can say more. Indeed, we have (2.5) together with convergence of all the moments (see Billingsley [2, p. 391]). Therefore, in particular,

$$
\frac{E\left[\bar{M}_{i}^{4}\right]}{(\sqrt{n / 16})^{4}} \rightarrow E\left[\mathcal{N}(0,1)^{4}\right], \quad n \rightarrow \infty,
$$

i.e. $E\left[\bar{M}_{i}^{4}\right]=O\left(n^{2}\right)$. This completes the proof of Theorem 1.1 (upper bound).

\section{Proof of Theorem 1.1, LOWER Bound}

We will show that for each $\epsilon>0$,

$$
P(\pi \leq \sigma)=\Omega\left((\alpha-\epsilon)^{n}\right),
$$

where

$$
\alpha=\sqrt[11]{\frac{25497938851324213335}{22 !}}=0.70879 \ldots
$$

Introduce $\pi^{*}$ ( $\sigma^{*}$ resp.), the permutation $\pi$ ( $\sigma$ resp.) with the element $n$ deleted. More generally, for $k \leq n, \pi^{k *}\left(\sigma^{k *}\right.$ resp.) is the permutation of $[n-k]$ obtained from $\pi$ ( $\sigma$ resp.) by deletion of the $k$ largest elements, $n, n-1, \ldots, n-k+1$. The key to the proof is the following.

Lemma 3.1. Let $k \in[n]$. If every northeast submatrix of $M(\pi, \sigma)$ with at most $k$ rows contains at least as many crosses as balls, and $\pi^{k *} \leq \sigma^{k *}$, then $\pi \leq \sigma$.

Before proceeding with the proof, we introduce one more bit of notation. Let $G_{n}$ be the empty $n \times n$ grid depicted in the $M(\cdot)$ 's of Figure 2, Figure 66 is a depiction of $G_{5}$ and an emboldened northeast-corner $3 \times 4$ subgrid of it, denoted by $C$. If $C$ is any subgrid of $G_{n}$, then $M(\cdot \mid C)$ denotes the submatrix of $M(\cdot)$ that "sits" on $C$. To repeat, the $(0,1)$-matrix criterion says that $\pi \leq \sigma$ if and only if for each northeast-corner subgrid $C$ of $G_{n}$, we have at least as many crosses as balls in $M(\pi, \sigma \mid C)$.

Proof. By the assumption, it suffices to show that the balls do not outnumber the crosses in $M(\pi, \sigma \mid C)$ for every such subgrid $C$ with strictly more than $k$ rows. Consider any such $C$. Let $C^{(k)}$ denote the subgrid formed by the top $k$ rows of $C$. Given a submatrix $A$ of $M(\pi)$ (of $M(\sigma)$ resp.), let $|A|$ denote the number of columns in $A$ with a cross (a ball resp.). We need to show

$$
|M(\pi \mid C)| \geq|M(\sigma \mid C)| .
$$

By the assumption, we have $\left|M\left(\pi \mid C^{(k)}\right)\right| \geq\left|M\left(\sigma \mid C^{(k)}\right)\right|$. Write $\left|M\left(\pi \mid C^{(k)}\right)\right|=$ $\left|M\left(\sigma \mid C^{(k)}\right)\right|+\lambda, \lambda \geq 0$. We now delete the top $k$ rows from $M(\pi), M(\sigma)$ together with the $k$ columns that contain the top $k$ crosses in the case of $M(\pi)$ and the 


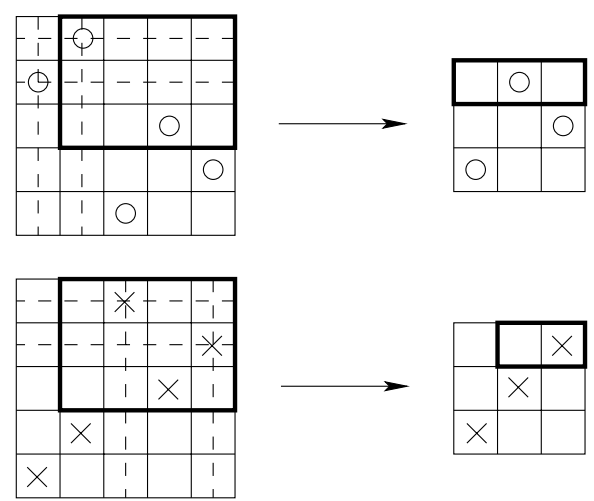

Figure 7. Deletion of 2 largest elements of $\pi, \sigma$, and its effect on $C$

$k$ columns that contain the top $k$ balls in the case of $M(\sigma)$. This produces the matrices $M\left(\pi^{k *}\right)$ and $M\left(\sigma^{k *}\right)$. In either case, we obtain the grid $G_{n-k}$ together with a new northeast subgrid: $C\left(\pi^{k *}\right)$ in the case of $M(\pi)$ and $C\left(\sigma^{k *}\right)$ in the case of $M(\sigma)$. Figure 7 is a graphical visualization of this deletion process in the special case $\pi=12534, \sigma=45132, k=2$ and $C$ the $3 \times 4$ northeast subgrid of $G_{5}$. We have emboldened $C$ in $M(\pi), M(\sigma)$, and the resulting $C\left(\pi^{2 *}\right), C\left(\sigma^{2 *}\right)$ in $M\left(\pi^{2 *}\right), M\left(\sigma^{2 *}\right)$ respectively.

Since we delete more columns in the case of $\pi$ than $\sigma$, note that $C\left(\pi^{k *}\right) \subseteq C\left(\sigma^{k *}\right)$ as northeast subgrids of $G_{n-k}$. In fact, these grids have the same number of rows, but $C\left(\pi^{k *}\right)$ has $\lambda$ fewer columns. Hence, as $\pi^{k *} \leq \sigma^{k *}$, we have

$$
\left|M\left(\pi^{k *} \mid C\left(\pi^{k *}\right)\right)\right| \geq\left|M\left(\sigma^{k *} \mid C\left(\pi^{k *}\right)\right)\right| \geq\left|M\left(\sigma^{k *} \mid C\left(\sigma^{k *}\right)\right)\right|-\lambda .
$$

So

$$
\begin{aligned}
|M(\pi \mid C)| & =\left|M\left(\pi \mid C^{(k)}\right)\right|+\left|M\left(\pi^{k *} \mid C\left(\pi^{k *}\right)\right)\right| \\
& =\left|M\left(\sigma \mid C^{(k)}\right)\right|+\lambda+\left|M\left(\pi^{k *} \mid C\left(\pi^{k *}\right)\right)\right| \\
& \geq\left|M\left(\sigma \mid C^{(k)}\right)\right|+\left|M\left(\sigma^{k *} \mid C\left(\sigma^{k *}\right)\right)\right| \\
& =|M(\sigma \mid C)|,
\end{aligned}
$$

which proves the lemma.

For each $k \leq n$, let $\mathcal{E}_{n, k}$ denote the event "every northeast submatrix of the top $k$ rows has at least as many crosses as balls". Then by Lemma 3.1,

$$
\{\pi \leq \sigma\} \supseteq \mathcal{E}_{n, k} \cap\left\{\pi^{k *} \leq \sigma^{k *}\right\} .
$$

Now the events $\mathcal{E}_{n, k}$ and $\left\{\pi^{k *} \leq \sigma^{k *}\right\}$ are independent! So we get

$$
P(\pi \leq \sigma) \geq P\left(\mathcal{E}_{n, k}\right) P\left(\pi^{k *} \leq \sigma^{k *}\right) .
$$

For the permutation $\pi$ ( $\sigma$ resp.) introduce $\ell_{i}(\pi)=\pi^{-1}(i)\left(\ell_{i}(\sigma)=\sigma^{-1}(i)\right.$ resp.), the index of a column that contains a cross (a ball resp.) at the intersection with row $i$. In terms of the $\ell_{i}(\cdot)$ 's, $\mathcal{E}_{n, k}$ is the event: for each integer $j \leq k$ and $m \leq n$, the number of $\ell_{n}(\pi), \ell_{n-1}(\pi), \ldots, \ell_{n-j+1}(\pi)$ that are $m$ at least is more than or equal to the number of $\ell_{n}(\sigma), \ell_{n-1}(\sigma), \ldots, \ell_{n-j+1}(\sigma)$ that are $m$ at least. We could 
have replaced an integer $m \leq n$ with a real number, which means that

$$
\mathcal{E}_{n, k}=\left\{(\pi, \sigma):(\ell(\pi), \ell(\sigma)) \in \mathcal{C}_{k}\right\},
$$

for some cone-shaped (Borel) set $\mathcal{C}_{k} \subset \mathbb{R}^{2 k}$; here $\boldsymbol{\ell}(\pi)=\left\{\ell_{n-i+1}(\pi)\right\}_{1 \leq i \leq k}$ and $\boldsymbol{\ell}(\sigma)=\left\{\ell_{n-i+1}(\sigma)\right\}_{1 \leq i \leq k}$.

Our task is to estimate sharply $P\left(\mathcal{E}_{n, k}\right)$ for a fixed $k$, and $n \rightarrow \infty$. Observe first that $\boldsymbol{\ell}(\pi)$ and $\boldsymbol{\ell}(\sigma)$ are independent, and each is uniformly distributed. For instance,

$$
P\left(\ell_{n}(\pi)=j_{1}, \ldots, \ell_{n-k+1}(\pi)=j_{k}\right)=\frac{1}{(n)_{k}}, \quad 1 \leq j_{1} \neq \cdots \neq j_{k} \leq n,
$$

where $(n)_{k}=n(n-1) \cdots(n-k+1)$. Since $(n)_{k} \sim n^{k}$ as $n \rightarrow \infty, \ell_{n}(\pi), \ldots$, $\ell_{n-k+1}(\pi)$ are almost independent $[n]$-uniforms for large $n$ and fixed $k$. Let us make this asymptotic reduction rigorous. Let $U$ be a $[0,1]$-uniform random variable, and let $U_{1}, \ldots, U_{n}$ be independent copies of $U$. Then each $\left\lceil n U_{i}\right\rceil$ is uniform on $[n]$, and it is easy to show that

$$
P\left(\left\lceil n U_{1}\right\rceil=i_{1}, \ldots,\left\lceil n U_{k}\right\rceil=i_{k} \mid\left\lceil n U_{1}\right\rceil \neq \cdots \neq\left\lceil n U_{k}\right\rceil\right)=\frac{1}{(n)_{k}} .
$$

In other words, $\left\{\ell_{n-i+1}(\pi)\right\}_{1 \leq i \leq k}$ has the same distribution as the random vector $\lceil n \mathbf{U}\rceil:=\left\{\left\lceil n U_{i}\right\rceil\right\}_{1 \leq i \leq k}$ conditioned on the event $\mathcal{A}_{n, k}=\left\{\left\lceil n U_{1}\right\rceil \neq \cdots \neq\left\lceil n U_{k}\right\rceil\right\}$. Analogously $\left\{\ell_{n-i+1}(\sigma)\right\}_{1 \leq i \leq k}$ is distributed as $\lceil n \mathbf{V}\rceil:=\left\{\left\lceil n V_{i}\right\rceil\right\}_{1 \leq i \leq k}$ conditioned on $\mathcal{B}_{n, k}=\left\{\left\lceil n V_{1}\right\rceil \neq \cdots \neq\left\lceil n V_{k}\right\rceil\right\}$, where $V_{1}, \ldots, V_{k}$ are independent $[0,1]$-uniforms, independent of $U_{1}, \ldots, U_{k}$. We will need yet another event $\mathcal{D}_{n, k}$ on which

$$
\min \left\{\min _{i \neq j}\left|U_{i}-U_{j}\right|, \min _{i \neq j}\left|V_{i}-V_{j}\right|, \min _{i, j}\left|U_{i}-V_{j}\right|\right\}>1 / n .
$$

Clearly on $\mathcal{D}_{n, k}$,

$$
(\lceil n \mathbf{U}\rceil,\lceil n \mathbf{V}\rceil) \in \mathcal{C}_{k} \Longleftrightarrow(\mathbf{U}, \mathbf{V}) \in \mathcal{C}_{k} ;
$$

here $\mathbf{U}:=\left\{U_{i}\right\}_{1 \leq i \leq k}, \mathbf{V}:=\left\{V_{i}\right\}_{1 \leq i \leq k}$. In addition $\mathcal{D}_{n, k} \subseteq \mathcal{A}_{n, k} \cap \mathcal{B}_{n, k}$, and

$$
P\left(\mathcal{D}_{n, k}^{c}\right) \leq 2 k^{2} P\left(\left|U_{1}-U_{2}\right| \leq 1 / n\right) \leq 4 k^{2} / n .
$$

Therefore

$$
\begin{aligned}
P\left(\mathcal{E}_{n, k}\right) & =P\left((\boldsymbol{\ell}(\pi), \boldsymbol{\ell}(\sigma)) \in \mathcal{C}_{k}\right) \\
& =\frac{P\left(\left\{(\lceil n \mathbf{U}\rceil,\lceil n \mathbf{V}\rceil) \in \mathcal{C}_{k}\right\} \cap\left\{\mathcal{A}_{n, k} \cap \mathcal{B}_{n, k}\right\}\right)}{P\left(\mathcal{A}_{n, k} \cap \mathcal{B}_{n, k}\right)} \\
& =\frac{P\left(\left\{(\lceil n \mathbf{U}\rceil,\lceil n \mathbf{V}\rceil) \in \mathcal{C}_{k}\right\} \cap \mathcal{D}_{n, k}\right)+O\left(P\left(\mathcal{D}_{n, k}^{c}\right)\right)}{1-O\left(P\left(\mathcal{D}_{n, k}^{c}\right)\right)} \\
& =\frac{P\left((\mathbf{U}, \mathbf{V}) \in \mathcal{C}_{k}\right)+O\left(k^{2} / n\right)}{1-O\left(k^{2} / n\right)} \\
& =Q_{k}+O\left(k^{2} / n\right),
\end{aligned}
$$

where $Q_{k}=P\left((\mathbf{U}, \mathbf{V}) \in \mathcal{C}_{k}\right)$. Let us write $P_{n}=P(\pi \leq \sigma)$. Using (3.1) and the last estimate, we obtain then

$$
P_{n} \geq Q_{k} P_{n-k}\left(1+O\left(k^{2} / n\right)\right)=Q_{k} P_{n-k} \exp \left(O\left(k^{2} / n\right)\right), \quad n>k .
$$


Iterating this inequality $\lfloor n / k\rfloor$ times gives

$$
P_{n} \geq Q_{k}^{\lfloor n / k\rfloor} P_{n-\lfloor n / k\rfloor k} \exp \left[\sum_{j=0}^{\lfloor n / k\rfloor-1} O\left(\frac{k^{2}}{n-j k}\right)\right] .
$$

Since the sum in the exponent is of order $O\left(k^{2} \log n\right)$, we get

$$
\liminf \sqrt[n]{P_{n}} \geq \sqrt[k]{Q_{k}}, \quad \forall k \geq 1 .
$$

Thus

$$
\liminf \sqrt[n]{P_{n}} \geq \sup _{k} \sqrt[k]{Q_{k}}
$$

Therefore, for each $k$ and $\epsilon \in\left(0, \sqrt[k]{Q_{k}}\right)$, we have

$$
P_{n}=\Omega\left(\left(\sqrt[k]{Q_{k}}-\epsilon\right)^{n}\right)
$$

Next

Lemma 3.2. As a function of $k, Q_{k}$ is supermultiplicative, i.e. $Q_{k_{1}+k_{2}} \geq Q_{k_{1}} Q_{k_{2}}$ for all $k_{1}, k_{2} \geq 1$. Consequently there exists $\lim _{k \rightarrow \infty} \sqrt[k]{Q_{k}}$, and moreover,

$$
\lim _{k \rightarrow \infty} \sqrt[k]{Q_{k}}=\sup _{k \geq 1} \sqrt[k]{Q_{k}}
$$

Thus we expect that our lower bound would probably improve as $k$ increases.

Proof. $Q_{k}$ is the probability of the event $E_{k}=\left\{\left(\mathbf{U}^{(k)}, \mathbf{V}^{(k)}\right) \in \mathcal{C}_{k}\right\}$; here $\mathbf{U}^{(k)}:=$ $\left\{U_{i}\right\}_{1 \leq i \leq k}, \mathbf{V}^{(k)}:=\left\{V_{i}\right\}_{1 \leq i \leq k}$. Explicitly, for each $j \leq k$ and each $c \in[0,1]$, the number of $U_{1}, \ldots, U_{j}$ not exceeding $c$ is at most the number of $V_{1}, \ldots, V_{j}$ not exceeding $c$. So $Q_{k_{1}+k_{2}}=P\left(E_{k_{1}+k_{2}}\right), Q_{k_{1}}=P\left(E_{k_{1}}\right)$, while $Q_{k_{2}}=P\left(E_{k_{2}}\right)=$ $P\left(E_{k_{2}}^{*}\right)$. Here the event $E_{k_{2}}^{*}$ means that for each $j^{*} \leq k_{2}$ and each $c \in[0,1]$, the number of $U_{i}, i=k_{1}+1, \ldots, k_{1}+j^{*}$, not exceeding $c$ is at most the number of $V_{i}$, $i=k_{1}+1, \ldots, k_{1}+j^{*}$, not exceeding $c$. The events $E_{k_{1}}$ and $E_{k_{2}}^{*}$ are independent. Consider the intersection of $E_{k_{1}}$ and $E_{k_{2}}^{*}$. There are two cases:

1) $j \leq k_{1}$. Then the number of $U_{i}, i \leq j$ not exceeding $c$ is at most the number of $V_{i}, i \leq j$ not exceeding $c$, as $E_{k_{1}}$ holds.

2) $k_{1}<j \leq k_{1}+k_{2}$. Then the number of $U_{i}, i \leq j$, not exceeding $c$ is at most the number of $V_{i}, i \leq k_{1}$ not exceeding $c$ (as $E_{k_{1}}$ holds), plus the number of $V_{i}, k_{1}<i \leq j$, not exceeding $c$ (as $E_{k_{2}}^{*}$ holds). The total number of these $V_{i}$ is the number of all $V_{i}, i \leq j$, that are at most $c, c \in[0,1]$.

So $E_{k_{1}+k_{2}} \supseteq E_{k_{1}} \cap E_{k_{2}}^{*}$, and we get $Q_{k_{1}+k_{2}} \geq Q_{k_{1}} Q_{k_{2}}$. The rest of the statement follows from a well-known result about super(sub)multiplicative sequences (see Pólya and Szegö [23, p. 23, ex. 98]).

Given $1 \leq j \leq i \leq k$, let $U_{i, j}\left(V_{i, j}\right.$ resp.) denote the $j$-th element in the increasing rearrangement of $U_{1}, \ldots, U_{i}\left(V_{1}, \ldots, V_{i}\right.$ resp.). Then, to put it another way, $Q_{k}$ is the probability that the $k$ Ehresmann conditions are met by the independent $k$ dimensional random vectors $\mathbf{U}$ and $\mathbf{V}$, both of which have independent entries. That is, we check $U_{i, j}>V_{i, j}$ for each $1 \leq j \leq i \leq k$ by performing elementwise 
comparisons in the following tableaux:

$\begin{array}{ccccc}U_{k, 1} & U_{k, 2} & U_{k, 3} & \cdots & U_{k, k} \\ \vdots & \vdots & \vdots & . \cdot & \\ U_{3,1} & U_{3,2} & U_{3,3} & & \\ U_{2,1} & U_{2,2} & & & \\ U_{1,1} & & & & \end{array}$

$\begin{array}{ccccc}V_{k, 1} & V_{k, 2} & V_{k, 3} & \cdots & V_{k, k} \\ \vdots & \vdots & \vdots & . \cdot & \\ V_{3,1} & V_{3,2} & V_{3,3} & & \\ V_{2,1} & V_{2,2} & & & \\ V_{1,1} & & & & \end{array}$.

What's left is to explain how we determined $\alpha=0.70879 \ldots$.

It should be clear that whether or not $\left(\mathbf{U}^{(k)}, \mathbf{V}^{(k)}\right)$ is in $\mathcal{C}_{k}$ depends only on the size ordering of $U_{1}, \ldots, U_{k}, V_{1}, \ldots, V_{k}$. There are $(2 k)$ ! possible orderings, all being equally likely. Thus $Q_{k}=N_{k} /(2 k)$ !, where $N_{k}$ is the number of these linear orderings satisfying this modified Ehresmann criterion. Since the best constant in the lower exponential bound is probably $\lim _{k \rightarrow \infty} \sqrt[k]{Q_{k}}$, our task was to compute $N_{k}$ for $k$ as large as our computer could handle ("probably", because we do not know for certain that $\sqrt[k]{Q_{k}}$ increases with $k$ ).

Here is how $N_{k}$ was tabulated. Recursively, suppose we have determined all $N_{k-1}$ orderings of $x_{1}, \ldots, x_{k-1}, y_{1}, \ldots, y_{k-1}$ such that $\left(\mathbf{x}^{(k-1)}, \mathbf{y}^{(k-1)}\right) \in \mathcal{C}_{k-1}$. Each such ordering can be assigned a 2(k-1)-long sequence of 0's and 1's, 0's for $x_{i}$ 's and 1's for $y_{j}$ 's, $1 \leq i, j \leq k-1$. Each such sequence meets the ballot-theorem condition: as we read it from left to right the number of 1's never falls below the number of 0 's. We also record the multiplicity of each sequence, which is the number of times it is encountered in the list of all $N_{k-1}$ orderings. The knowledge of all $2(k-1)$-long ballot-sequences together with their multiplicities is all we need to compile the list of all $2 k$-long ballot-sequences with their respective multiplicities.

For $k=1$, there is only one ballot-sequence to consider, namely 10 , and its multiplicity is 1 . So $N_{1}=1$, and

$$
Q_{1}=1 / 2 !
$$

Passing to $k=2$, we must count the number of ways to insert 1 and 0 into 10 so that we get a 4-long ballot-sequence of two 0's and two 1's. Inserting 1 at the beginning, giving 110 , we can insert 0 into positions 2,3 or 4 , producing three ballot-sequences

$$
\text { 1010, 1100, 1100, }
$$

respectively. (Inserting 0 into position 1 would have resulted in 0110 , which is not a ballot-sequence.) Similarly, inserting 1 into position 2, we get 110, and inserting 0 under the ballot condition gives three ballot-sequences

$$
1010, \quad 1100, \quad 1100 .
$$

Finally, inserting 1 at the end, giving 101, we can only insert 0 at the end, obtaining one ballot-sequence

$$
1010 .
$$

Hence, starting from the ballot-sequence 10 of multiplicity 1, we have obtained two 4-long ballot-sequences, 1010 of multiplicity 3 and 1100 of multiplicity 4 . Therefore $N_{2}=3+4=7$, and

$$
Q_{2}=7 / 4 !
$$

Pass to $k=3$. Sequentially we insert 1 in each of 5 positions in the ballot-sequence 1010, and then determine all positions for the new 0 which would result in a 6 -long ballot-sequence. While doing this we keep track of how many times each 6-long 
ballot-sequence is encountered. Multiplying these numbers by 3 , the multiplicity of 1010, we obtain a list of 6 -long ballot-sequences spawned by 1010 with the number of their occurrences. We do the same with the second sequence 1100. Adding the numbers of occurrences of each 6-long ballot-sequence for 1010 and 1100, we arrive at the following list of five 6 -long ballot-sequences with their respective multiplicities:

$$
\begin{aligned}
& 111000: 36, \\
& 110100: 32, \\
& 110010: 24, \\
& 101100: 24, \\
& 101010: 19 .
\end{aligned}
$$

Therefore $N_{3}=36+32+24+24+19=135$, and

$$
Q_{3}=135 / 6 \text { !. }
$$

The younger coauthor wrote a computer program for this algorithm. Pushed to its limit, the computer delivered the following table:

\begin{tabular}{r|r|l|c}
$k$ & $N_{k}=(2 k) ! Q_{k}$ & $Q_{k}=N_{k} /(2 k) !$ & $\sqrt[k]{Q_{k}}$ \\
\hline 1 & 1 & $0.50000 \ldots$ & $0.50000 \ldots$ \\
2 & 7 & $0.29166 \ldots$ & $0.54006 \ldots$ \\
3 & 135 & $0.18750 \ldots$ & $0.57235 \ldots$ \\
4 & 5193 & $0.12879 \ldots$ & $0.59906 \ldots$ \\
5 & 336825 & $0.09281 \ldots$ & $0.62162 \ldots$ \\
6 & 33229775 & $0.06937 \ldots$ & $0.64101 \ldots$ \\
7 & 4651153871 & $0.05335 \ldots$ & $0.65790 \ldots$ \\
9 & 878527273745 & $0.04198 \ldots$ & $0.67280 \ldots$ \\
10 & 215641280371953 & $0.03368 \ldots$ & $0.68608 \ldots$ \\
11 & 2549791817776602071 & $0.02745 \ldots$ & $0.69800 \ldots$ \\
& 2538851324213335 & $0.02268 \ldots$ & $0.70879 \ldots$
\end{tabular}

Combining (3.2) and the value of $\sqrt[11]{Q_{11}}$ in this table, we see that for each $\epsilon>0$,

$$
P_{n}=\Omega\left(\left(\sqrt[11]{Q_{11}}-\epsilon\right)^{n}\right)=\Omega\left((0.708 \ldots-\epsilon)^{n}\right) .
$$

The numbers $\sqrt[k]{Q_{k}}$ increase steadily for $k<12$, so at this moment we would not rule out the tantalizing possibility that $\sqrt[k]{Q_{k}} \rightarrow 1$ as $k \rightarrow \infty$. Determination of the actual limit is a challenging open problem. The proof just given only involves mention of the $(0,1)$-matrix criterion, but it was the Ehresmann criterion that actually inspired our initial insights.

\section{WEAK ORDER, PRELIMINARIES}

Recall that $\pi$ precedes $\sigma$ in the weak order $(\pi \preceq \sigma)$ if and only if there is a chain $\sigma=\omega_{1} \rightarrow \cdots \rightarrow \omega_{s}=\pi$ where each $\omega_{t}$ is a simple reduction of $\omega_{t-1}$, i.e. obtained by transposing two adjacent elements $\omega_{t-1}(i), \omega_{t-1}(i+1)$ such that $\omega_{t-1}(i)>\omega_{t-1}(i+1)$. Clearly the weak order is more restrictive than the Bruhat order, so that $\pi \preceq \sigma$ implies $\pi \leq \sigma$. In particular, $P(\pi \preceq \sigma) \leq P(\pi \leq \sigma)$; hence (Theorem 1.1) $P(\pi \preceq \sigma)=O\left(n^{-2}\right)$. We will show that, in fact, this probability is exponentially small. The proof is based on an inversion set criterion for $\pi \preceq \sigma$ implicit in [1, pp. 135-139]. 
Lemma 4.1. Given $\omega \in S_{n}$, introduce the set of noninversions of $\omega$ :

$$
E(\omega):=\left\{(i, j): i<j, \omega^{-1}(i)<\omega^{-1}(j)\right\} .
$$

$\pi \preceq \sigma$ if and only if $E(\pi) \supseteq E(\sigma)$.

Proof. Assume $\pi \preceq \sigma$. Then there exists a chain of simple reductions $\omega_{t}, 1 \leq t \leq s$, connecting $\sigma=\omega_{1}$ and $\pi=\omega_{s}$. By the definition of a simple reduction, for each $t>1$ there is $i=i_{t}<n$ such that $E\left(\omega_{t}\right)=E\left(\omega_{t-1}\right) \cup\left\{\left(\omega_{t}(i), \omega_{t}(i+1)\right)\right\}$, where $\omega_{t}(i)=\omega_{t-1}(i+1), \omega_{t}(i+1)=\omega_{t-1}(i)$, and $\omega_{t-1}(i)>\omega_{t-1}(i+1)$. So the set $E\left(\omega_{t}\right)$ increases with $t$; hence $E(\pi) \supseteq E(\sigma)$.

Conversely, suppose $E(\pi) \supseteq E(\sigma)$. Since a permutation $\omega$ is uniquely determined by its $E(\omega)$, we may assume $E(\pi) \supsetneq E(\sigma)$.

Claim. If $E(\pi) \supsetneq E(\sigma)$, then there exists $u<v \leq n$ such that $(v, u)$ is an adjacent inversion of $\sigma$, but $(u, v) \in E(\pi)$.

Assuming validity of the claim, we ascertain existence of an adjacent inversion $(v, u)$ in $\sigma$ with $(u, v) \in E(\pi)$. Interchanging the adjacent elements $u$ and $v$ in $\sigma=\omega_{1}$, we obtain a simple reduction $\omega_{2}$, with $E\left(\omega_{1}\right) \subset E\left(\omega_{2}\right) \subseteq E(\pi)$. If $E\left(\omega_{2}\right)=$ $E(\pi)$, then $\omega_{2}=\pi$, and we stop. Otherwise we determine $\omega_{3}$, a simple reduction of $\omega_{2}$, with $E\left(\omega_{2}\right) \subset E\left(\omega_{3}\right) \subseteq E(\pi)$ and so on. Eventually we determine a chain of simple reductions connecting $\sigma$ and $\pi$, which proves that $\pi \preceq \sigma$.

Proof of claim. The claim is obvious for $n=1,2$. Assume inductively that the claim holds for permutations of length $n-1 \geq 2$. Let $\pi, \sigma \in S_{n}$ and $E(\pi) \supsetneq E(\sigma)$. As in the proof of Theorem 1.1, let $\ell_{n}(\pi)=\pi^{-1}(n), \ell_{n}(\sigma)=\sigma^{-1}(n)$, and $\pi^{*}$, $\sigma^{*}$ are obtained by deletion of $n$ from $\pi$ and $\sigma$. Since $E(\pi) \supseteq E(\sigma)$, we have $E\left(\pi^{*}\right) \supseteq E\left(\sigma^{*}\right)$. Suppose first that $E\left(\pi^{*}\right)=E\left(\sigma^{*}\right)$. Then $\pi^{*}=\sigma^{*}$, and as $E(\pi) \supsetneq E(\sigma)$, we must have $\ell_{n}(\pi)>\ell_{n}(\sigma)$, i.e. $\ell_{n}(\sigma)<n$. Setting $v=n$ and $u=\sigma\left(\ell_{n}(\sigma)+1\right)$, we obtain an adjacent inversion $(v, u)$ in $\sigma$ with $(u, v) \in E(\pi)$.

Alternatively, $E\left(\pi^{*}\right) \supsetneq E\left(\sigma^{*}\right)$. By inductive hypothesis, there exists $u<v \leq$ $n-1$ such that $(v, u)$ is an adjacent inversion of $\sigma^{*}$, but $(u, v) \in E\left(\pi^{*}\right)$. Now insert $n$ back into $\pi^{*}, \sigma^{*}$, recovering $\pi$ and $\sigma$. If $n$ sits to the right of $u$ or to the left of $v$ in $\sigma$, then $(v, u)$ is still an adjacent inversion of $\sigma$. Otherwise $n$ is sandwiched between $v$ on the left and $u$ on the right. Therefore $(n, u)$ is an adjacent inversion in $\sigma$. On the other hand $(v, n) \in E(\sigma)$, so since $E(\pi) \supseteq E(\sigma)$, we have $(v, n) \in E(\pi)$ also. Hence, the triple $(u, v, n)$ are in exactly this order (not necessarily adjacent) in $\pi$. Therefore the adjacent inversion $(n, u)$ in $\sigma$ is such that $(u, n) \in E(\pi)$, and this proves the inductive step.

Denote by $\bar{\omega}$ the permutation $\omega$ reversed in rank. For example, with $\omega=13254$ we have $\bar{\omega}=53412$. Then it is easy to see that

$$
E(\pi) \supseteq E(\sigma) \Longleftrightarrow E(\bar{\pi}) \subseteq E(\bar{\sigma}) .
$$

By Lemma 4.1, these statements are equivalent to

$$
\pi \preceq \sigma \Longleftrightarrow \bar{\sigma} \preceq \bar{\pi} .
$$

We immediately obtain the following corollary to Lemma 4.1;

Corollary 1. For $\omega \in S_{n}$, define

$$
E_{i}(\omega):=\{j<i:(j, i) \in E(\omega)\}, \quad 1 \leq i \leq n .
$$


Then

and consequently

$$
E(\omega)=\bigsqcup_{i=1}^{n}\left\{(j, i): j \in E_{i}(\omega)\right\}
$$

$$
\pi \preceq \sigma \Longleftrightarrow E(\pi) \supseteq E(\sigma) \Longleftrightarrow E_{i}(\pi) \supseteq E_{i}(\sigma), \quad \forall i \leq n .
$$

Next,

Lemma 4.2. For $\pi, \sigma \in S_{n}$ selected independently and uniformly at random, let $P_{n}^{*}:=P(\pi \preceq \sigma)$. $P_{n}^{*}$ is submultiplicative, i.e. for all $n_{1}, n_{2} \geq 1$,

$$
P_{n_{1}+n_{2}}^{*} \leq P_{n_{1}}^{*} P_{n_{2}}^{*}
$$

Consequently there exists $\lim _{n \rightarrow \infty} \sqrt[n]{P_{n}^{*}}=\inf _{n \geq 1} \sqrt[n]{P_{n}^{*}}$.

Proof. Let $\pi, \sigma$ be two permutations of $\left[n_{1}+n_{2}\right]$. Then $\pi \preceq \sigma$ if and only if

$$
E_{i}(\pi) \supseteq E_{i}(\sigma), \quad 1 \leq i \leq n_{1}+n_{2} .
$$

Using these conditions for $i \leq n_{1}$, we see that

$$
\pi\left[1,2, \ldots, n_{1}\right] \preceq \sigma\left[1,2, \ldots, n_{1}\right] .
$$

Here $\pi\left[1,2, \ldots, n_{1}\right]$, say, is what is left of the permutation $\pi$ when the elements $n_{1}+1, \ldots, n_{1}+n_{2}$ are deleted.

Likewise, $\pi \preceq \sigma$ if and only if

$$
E_{i}(\bar{\pi}) \subseteq E_{i}(\bar{\sigma}), \quad 1 \leq i \leq n_{1}+n_{2} .
$$

Using these conditions for $i \leq n_{2}$, we see that

$$
\pi\left[n_{1}+1, \ldots, n_{1}+n_{2}\right] \preceq \sigma\left[n_{1}+1, \ldots, n_{1}+n_{2}\right] .
$$

Now, since $\pi$ and $\sigma$ are uniformly random and mutually independent, so are the four permutations

$$
\pi\left[1, \ldots, n_{1}\right], \quad \pi\left[n_{1}+1, \ldots, n_{1}+n_{2}\right], \quad \sigma\left[1, \ldots, n_{1}\right], \quad \sigma\left[n_{1}+1, \ldots, n_{1}+n_{2}\right] .
$$

Hence,

$$
\begin{aligned}
P(\pi \preceq \sigma) \leq P\left(\pi\left[1, \ldots, n_{1}\right] \preceq \sigma\left[1, \ldots, n_{1}\right]\right) & \\
& \times P\left(\pi\left[n_{1}+1, \ldots, n_{1}+n_{2}\right] \preceq \sigma\left[n_{1}+1, \ldots, n_{1}+n_{2}\right]\right),
\end{aligned}
$$

so that

$$
P_{n_{1}+n_{2}}^{*} \leq P_{n_{1}}^{*} P_{n_{2}}^{*} .
$$

\section{Proof of Theorem 1.2, Upper Bound}

We will show that for each $\epsilon>0$,

$$
P_{n}^{*}=O\left((\beta+\epsilon)^{n}\right),
$$

where

$$
\beta=\sqrt[6]{\frac{1065317}{12 !}}=0.36129 \ldots
$$

The proof of this upper bound for $P_{n}^{*}$ parallels the proof of the lower bound for $P_{n}$ in Theorem 1.1. As in that proof, given $k \geq 1$, let $\pi^{k *}$ and $\sigma^{k *}$ be obtained by deletion of the elements $n, \ldots, n-k+1$ from $\pi$ and $\sigma$, and let $\ell_{i}(\pi)=\pi^{-1}(i)$, $\ell_{i}(\sigma)=\sigma^{-1}(i), n-k+1 \leq i \leq n$. In the notation of the proof of Lemma 4.2, 
$\pi^{k *}=\pi[1, \ldots, n-k]$ and $\sigma^{k *}=\sigma[1, \ldots, n-k]$, and we saw that $\pi^{k *} \preceq \sigma^{k *}$ if $\pi \preceq \sigma$. Our task is to find the conditions these $\ell_{i}(\cdot)$ 's must satisfy if $\pi \preceq \sigma$ holds.

To start, notice that

$$
\pi \preceq \sigma \Longrightarrow\left|E_{n}(\pi)\right| \geq\left|E_{n}(\sigma)\right| \Longleftrightarrow \ell_{n}(\pi) \geq \ell_{n}(\sigma) .
$$

Next

$$
\pi \preceq \sigma \Longrightarrow \pi^{*} \preceq \sigma^{*} \Longrightarrow \ell_{n-1}(\pi) \geq \ell_{n-1}\left(\pi^{*}\right) \geq \ell_{n-1}\left(\sigma^{*}\right) \geq \ell_{n-1}(\sigma)-1,
$$

as deletion of $n$ from $\pi, \sigma$ decreases the location of $n-1$ in each permutation by at most one. In general, for $0<j<k$ we get

$$
\pi \preceq \sigma \Longrightarrow \pi^{j *} \preceq \sigma^{j *} \Longrightarrow \ell_{n-j}(\pi) \geq \ell_{n-j}(\sigma)-j .
$$

So, introducing $\boldsymbol{\ell}(\pi)=\left\{\ell_{n-i+1}(\pi)\right\}_{1 \leq i \leq k}$ and $\boldsymbol{\ell}(\sigma)=\left\{\ell_{n-i+1}(\sigma)\right\}_{1 \leq i \leq k}$,

$$
\begin{gathered}
\{\pi \preceq \sigma\} \subseteq\left\{(\boldsymbol{\ell}(\pi), \ell(\sigma)) \in \mathcal{S}_{k}\right\}, \\
\mathcal{S}_{k}:=\left\{(\mathbf{x}, \mathbf{y}) \in \mathbb{R}^{2 k}: x_{j} \geq y_{j}-j+1,1 \leq j \leq k\right\} .
\end{gathered}
$$

In addition, on $\{\pi \preceq \sigma\}$, every pair of elements, which form an inversion in $\pi$, also form an inversion in $\sigma$. Applying this to the elements $n-k+1, \ldots, n$, we have then

$$
\begin{gathered}
\{\pi \preceq \sigma\} \subseteq\left\{(\boldsymbol{\ell}(\pi), \ell(\sigma)) \in \mathcal{T}_{k}\right\}, \\
\mathcal{T}_{k}:=\left\{(\mathbf{x}, \mathbf{y}) \in \mathbb{R}^{2 k}: \forall 1 \leq i<j \leq k, x_{i}<x_{j} \Longrightarrow y_{i}<y_{j}\right\} .
\end{gathered}
$$

Combining (5.1) and (5.2), we get

$$
\{\pi \preceq \sigma\} \subseteq\left\{(\ell(\pi), \ell(\sigma)) \in \mathcal{S}_{k} \cap \mathcal{T}_{k}\right\} \cap\left\{\pi^{k *} \preceq \sigma^{k *}\right\} .
$$

So, since the two events on the right are independent,

$$
P_{n}^{*} \leq P\left((\ell(\pi), \ell(\sigma)) \in \mathcal{S}_{k} \cap \mathcal{T}_{k}\right) P_{n-k}^{*} .
$$

It remains to estimate $P\left((\ell(\pi), \ell(\sigma)) \in \mathcal{S}_{k} \cap \mathcal{T}_{k}\right)$. As in the proof of Theorem 1.1 (lower bound), we observe that $(\boldsymbol{\ell}(\pi), \boldsymbol{\ell}(\sigma))$ is equal in distribution to $(\lceil n \mathbf{U}\rceil,\lceil n \mathbf{V}\rceil)$, conditioned on

$$
\mathcal{C}_{n, k}:=\mathcal{A}_{n, k} \cap \mathcal{B}_{n, k}=\left\{\left\lceil n U_{1}\right\rceil \neq \cdots \neq\left\lceil n U_{k}\right\rceil\right\} \cap\left\{\left\lceil n V_{1}\right\rceil \neq \cdots \neq\left\lceil n V_{k}\right\rceil\right\} .
$$

Here $U_{1}, \ldots, U_{k}, V_{1}, \ldots, V_{k}$ are independent $[0,1]$-uniforms. Then

$$
P\left((\boldsymbol{\ell}(\pi), \boldsymbol{\ell}(\sigma)) \in \mathcal{S}_{k} \cap \mathcal{T}_{k}\right)=\frac{P\left(\left\{(\lceil n \mathbf{U}\rceil,\lceil n \mathbf{V}\rceil) \in \mathcal{S}_{k} \cap \mathcal{T}_{k}\right\} \cap \mathcal{C}_{n, k}\right)}{P\left(\mathcal{C}_{n, k}\right)} .
$$

Introduce the event $\tilde{\mathcal{D}}_{n, k}$ on which

$$
\min \left\{\min _{i \neq j}\left|U_{i}-U_{j}\right|, \min _{i \neq j}\left|V_{i}-V_{j}\right|, \min _{i, j}\left|U_{i}-V_{j}\right|, k^{-1} \min _{j}\left|U_{j}-V_{j}\right|\right\}>1 / n .
$$

Certainly $\tilde{\mathcal{D}}_{n, k} \subseteq \mathcal{C}_{n, k}$ and, thanks to the factor $1 / k$ by $\min _{j}\left|U_{j}-V_{j}\right|$, on $\tilde{\mathcal{D}}_{n, k}$,

$$
\left\lceil n U_{j}\right\rceil \geq\left\lceil n V_{j}\right\rceil-j+1 \Longrightarrow U_{j} \geq V_{j}-k / n \Longrightarrow U_{j}>V_{j} .
$$

Therefore, on $\tilde{\mathcal{D}}_{n, k}$,

$$
\begin{gathered}
(\lceil n \mathbf{U}\rceil,\lceil n \mathbf{V}\rceil) \in \mathcal{S}_{k} \cap \mathcal{T}_{k} \Longrightarrow(\mathbf{U}, \mathbf{V}) \in \tilde{\mathcal{S}}_{k} \cap \mathcal{T}_{k}, \\
\tilde{\mathcal{S}}_{k}:=\left\{(\mathbf{x}, \mathbf{y}) \in \mathbb{R}^{2 k}: x_{j}>y_{j}, 1 \leq j \leq k\right\} .
\end{gathered}
$$


Clearly $\tilde{\mathcal{S}}_{k} \cap \mathcal{T}_{k}$ is a cone-shaped subset of $\mathbb{R}^{2 k}$. In addition, $P\left(\tilde{\mathcal{D}}_{n, k}^{c}\right)=O\left(k^{2} / n\right)$. Hence

$$
\begin{aligned}
P\left((\boldsymbol{\ell}(\pi), \ell(\sigma)) \in \mathcal{S}_{k} \cap \mathcal{T}_{k}\right) & \leq \frac{P\left((\mathbf{U}, \mathbf{V}) \in \tilde{\mathcal{S}}_{k} \cap \mathcal{T}_{k}\right)+O\left(P\left(\tilde{\mathcal{D}}_{n, k}^{c}\right)\right)}{1-O\left(P\left(\tilde{\mathcal{D}}_{n, k}^{c}\right)\right)} \\
& =Q_{k}^{*}\left(1+O\left(k^{2} / n\right)\right), \quad Q_{k}^{*}:=P\left((\mathbf{U}, \mathbf{V}) \in \tilde{\mathcal{S}}_{k} \cap \mathcal{T}_{k}\right) .
\end{aligned}
$$

This and (5.3) imply

$$
P_{n}^{*} \leq Q_{k}^{*} P_{n-k}^{*} \exp \left(O\left(k^{2} / n\right)\right) .
$$

Hence, as in the proof of Theorem 1.1 (lower bound),

$$
\limsup \sqrt[n]{P_{n}^{*}} \leq \sqrt[k]{Q_{k}^{*}}, \quad \forall k \geq 1,
$$

and so

$$
P_{n}^{*}=O\left(\left(\sqrt[k]{Q_{k}^{*}}+\epsilon\right)^{n}\right), \quad k \geq 1, \epsilon>0 .
$$

Furthermore, from the definition of $Q_{k}^{*}$, it follows directly that $Q_{k}^{*}$ is submultiplicative; i.e.

$$
Q_{k_{1}+k_{2}}^{*} \leq Q_{k_{1}}^{*} Q_{k_{2}}^{*}, \quad k_{1}, k_{2} \geq 1 .
$$

Therefore ([23, p. 23, ex. 98] again)

$$
\lim _{k \rightarrow \infty} \sqrt[k]{Q_{k}^{*}}=\inf _{k \geq 1} \sqrt[k]{Q_{k}^{*}}
$$

So the further we can push tabulation of $Q_{k}^{*}$, the better our exponential upper bound for $P_{n}^{*}$ would probably be ("probably", because we do not have a proof that $\sqrt[k]{Q_{k}^{*}}$ decreases with $k$ ).

As in the case of $Q_{k}, Q_{k}^{*}=N_{k}^{*} /(2 k)$ !. Here, by the definition of the sets $\tilde{\mathcal{S}}_{k}$ and $\mathcal{T}_{k}, N_{k}^{*}$ is the total number of ways to order $x_{1}, \ldots, x_{k}, y_{1}, \ldots, y_{k}$ so that two conditions are met: (1) for each $j, x_{j}$ is to the right of $y_{j}$; (2) for all $i<j$, if $x_{i}$ is to the left of $x_{j}$, then $y_{i}$ is to the left of $y_{j}$.

It is instructive first to evaluate $N_{k}^{*}$ by hand for $k=1,2 . N_{1}^{*}=1$ as there is only one sequence, $y_{1} x_{1}$, meeting the conditions (1), (2). Passing to $N_{2}^{*}$, we must decide how to insert $y_{2}$ and $x_{2}$ into the sequence $y_{1} x_{1}$ in compliance with conditions (1), (2). First of all, $y_{2}$ has to precede $x_{2}$. If we insert $x_{2}$ at the beginning of $y_{1} x_{1}$, giving $x_{2} y_{1} x_{1}$, then we can only insert $y_{2}$ at the beginning of this triple, giving

$$
y_{2} x_{2} y_{1} x_{1} .
$$

Alternatively, inserting $x_{2}$ in the middle of $y_{1} x_{1}$, we have 2 possibilities for insertion of $y_{2}$, and we get two admissible orderings,

$$
y_{2} y_{1} x_{2} x_{1}, \quad y_{1} y_{2} x_{2} x_{1} .
$$

Finally, insertion of $x_{2}$ at the end of $y_{1} x_{1}$ brings the condition (2) into play as we now have $x_{1}$ preceding $x_{2}$, and so $y_{1}$ must precede $y_{2}$. Consequently, we get two admissible orderings,

$$
y_{1} y_{2} x_{1} x_{2}, \quad y_{1} x_{1} y_{2} x_{2}
$$

Hence $N_{2}^{*}=1+2+2=5$. Easy so far! However, passing to $k=3$ is considerably more time-consuming than it was for computation of $N_{3}$ in the proof of the lower bound in Theorem 1.1. There, once we had determined the $N_{2}$ admissible orderings, we could afford not to keep track of relative orderings of $x_{1}, \ldots, x_{k-1}$, and of $y_{1}, \ldots, y_{k-1}$, whence the coding by 1's and 0's. All we needed for passing from $k-1$ to $k$ was the list of all binary ballot-sequences of length $2(k-1)$ together with their 
multiplicities. Here the nature of the conditions (1), (2) does not allow lumping various sequences together, and we have to preserve the information of relative orderings of $x$ 's, and relative orderings of $y$ 's. This substantial complication seriously inhibits the computer's ability to compute $N_{k}^{*}$ for $k$ as large as in the case of $N_{k}$.

To get a feeling for how sharply the amount of computation increases for $k=3$, let us consider one of the $N_{2}^{*}=5$ admissible sequences, namely $y_{2} x_{2} y_{1} x_{1}$. As above, we write down all possible ways to insert $y_{3}$ and $x_{3}$ into this sequence so that (1) and (2) hold. Doing this, we produce the 10 sequences:

$$
\begin{array}{ll}
y_{3} x_{3} y_{2} x_{2} y_{1} x_{1}, & y_{3} y_{2} x_{3} x_{2} y_{1} x_{1}, \\
y_{2} y_{3} x_{3} x_{2} y_{1} x_{1}, & y_{2} y_{3} x_{2} x_{3} y_{1} x_{1}, \\
y_{2} x_{2} y_{3} x_{3} y_{1} x_{1}, & y_{2} y_{3} x_{2} y_{1} x_{3} x_{1}, \\
y_{2} x_{2} y_{3} y_{1} x_{3} x_{1}, & y_{2} x_{2} y_{1} y_{3} x_{3} x_{1}, \\
y_{2} x_{2} y_{1} y_{3} x_{1} x_{3}, & y_{2} x_{2} y_{1} x_{1} y_{3} x_{3} .
\end{array}
$$

We treat similarly the other four sequences from the $k=2$ case, eventually arriving at $N_{3}^{*}=55$. We wouldn't even think of computing $N_{4}^{*}$ by hand.

Once again the computer programming to the rescue! Here is the table produced by the computer after a substantial running time:

\begin{tabular}{r|r|l|c}
$k$ & $N_{k}^{*}=(2 k) ! Q_{k}^{*}$ & $Q_{k}^{*}=N_{k}^{*} /(2 k) !$ & $\sqrt[k]{Q_{k}^{*}}$ \\
\hline 1 & 1 & $0.50000 \ldots$ & $0.50000 \ldots$ \\
2 & 5 & $0.20833 \ldots$ & $0.45643 \ldots$ \\
3 & 55 & $0.07638 \ldots$ & $0.42430 \ldots$ \\
4 & 1023 & $0.02537 \ldots$ & $0.39910 \ldots$ \\
5 & 28207 & $0.00777 \ldots$ & $0.37854 \ldots$ \\
6 & 1065317 & $0.00222 \ldots$ & $0.36129 \ldots$
\end{tabular}

Using (5.4) for $k=6$, we get for each $\epsilon>0$,

$$
P_{n}^{*}=\left(\left(\sqrt[6]{Q_{6}^{*}}+\epsilon\right)^{n}\right)=\left((0.361 \ldots+\epsilon)^{n}\right) .
$$

\section{Proof of Theorem 1.2, LoWer Bound}

To bound $P(\pi \preceq \sigma)$ from below we will use the criterion

$$
\pi \preceq \sigma \Longleftrightarrow E_{i}(\pi) \supseteq E_{i}(\sigma), \quad \forall i \leq n .
$$

First of all,

Lemma 6.1. Let $i \in[n], B \subseteq[i-1]([0]=\emptyset)$. If $\pi \in S_{n}$ is chosen uniformly at random, then

$$
P\left(E_{i}(\pi) \supseteq B\right)=\frac{1}{|B|+1} .
$$

Proof. By the definition of $E_{i}(\pi)$,

$$
\left\{E_{i}(\pi) \supseteq B\right\}=\left\{\pi^{-1}(j)<\pi^{-1}(i), \forall j \in B\right\} .
$$

It remains to observe that $\pi^{-1}$ is also uniformly random. 
Lemma 6.1 implies the following key statement:

Lemma 6.2. Let $\pi, \sigma \in S_{n}$ be selected independently and uniformly at random. Then, for $i \in[n]$,

$$
P\left(E_{i}(\pi) \supseteq E_{i}(\sigma)\right)=H(i) / i, \quad H(i):=\sum_{j=1}^{i} \frac{1}{j} .
$$

Proof. By Lemma 6.1.

$$
\begin{aligned}
P\left(E_{i}(\pi) \supseteq E_{i}(\sigma)\right) & =\sum_{B \subseteq[i-1]} P\left(E_{i}(\pi) \supseteq B\right) P\left(E_{i}(\sigma)=B\right) \\
& =\sum_{B \subseteq[i-1]} \frac{P\left(E_{i}(\sigma)=B\right)}{|B|+1} \\
& =E\left[\frac{1}{\left|E_{i}(\sigma)\right|+1}\right] \\
& =\sum_{j=0}^{i-1} \frac{1}{i(j+1)}=\frac{H(i)}{i} .
\end{aligned}
$$

Note. In the second to last equality, we have used the fact that $\left|E_{i}(\sigma)\right|$ is distributed uniformly on $\{0,1, \ldots, i-1\}$. In addition, $\left|E_{1}(\sigma)\right|, \ldots,\left|E_{n}(\sigma)\right|$ are independent, a property we will use later. For completeness, here is a bijective proof of these facts. By induction, the numbers $\left|E_{i}(\sigma)\right|, i \leq t$, determine uniquely the relative ordering of elements $1, \ldots, t$ in the permutation $\sigma$. Hence the numbers $\left|E_{i}(\sigma)\right|$, $i \in[n]$, determine $\sigma$ uniquely. Since the range of $\left|E_{i}(\sigma)\right|$ is the set $\{0, \ldots, i-1\}$ of cardinality $i$, and $\left|S_{n}\right|=n$ !, it follows that the numbers $\left|E_{i}(\sigma)\right|, i \in[n]$, are uniformly distributed, and independent of each other.

Needless to say we are interested in $P(\pi \preceq \sigma)=P\left(\bigcap_{i=1}^{n}\left\{E_{i}(\pi) \supseteq E_{i}(\sigma)\right\}\right)$. Fortunately, the events $\left\{E_{i}(\pi) \supseteq E_{i}(\sigma)\right\}$ turn out to be positively correlated, and the product of the marginals $P\left(E_{i}(\pi) \supseteq E_{i}(\sigma)\right)$ bounds that probability from below.

Theorem 6.3. Let $\pi, \sigma \in S_{n}$ be selected independently and uniformly at random. Then

$$
P(\pi \preceq \sigma) \geq \prod_{i=1}^{n} P\left(E_{i}(\pi) \supseteq E_{i}(\sigma)\right)=\prod_{i=1}^{n} \frac{H(i)}{i} .
$$

Proof. First notice that, conditioning on $\sigma$ and using the independence of $\pi$ and $\sigma$,

$$
\begin{aligned}
P\left(E_{i}(\pi) \supseteq\right. & \left.E_{i}(\sigma), \forall i \leq n\right) \\
& =E\left[P\left(E_{i}(\pi) \supseteq E_{i}(\sigma), \forall i \leq n \mid \sigma\right)\right] \\
& =E\left[\left.P\left(E_{i}(\pi) \supseteq B_{i}, \forall i \leq n\right)\right|_{B_{i}=E_{i}(\sigma)}\right] .
\end{aligned}
$$

So our task is to bound $P\left(E_{i}(\pi) \supseteq B_{i}, \forall i \leq n\right)$, where these $B_{i}$ 's inherit the following property from the $E_{i}(\sigma)$ 's:

$$
i \in E_{j}(\sigma) \text { and } j \in E_{k}(\sigma) \Longrightarrow i \in E_{k}(\sigma) \text {. }
$$

Lemma 6.4. Let $n \geq 1$ be an integer, and let $B_{i} \subseteq[n], i=1, \ldots, n$, be such that $i \notin B_{i}$ and $i \in B_{j}, j \in B_{k} \Longrightarrow i \in B_{k}, \quad \forall i, j, k \in[n]$. 
Then, for $\pi \in S_{n}$ selected uniformly at random,

$$
P\left(E_{i}(\pi) \supseteq B_{i}, \forall i \leq n\right) \geq \prod_{i=1}^{n} \frac{1}{\left|B_{i}\right|+1} .
$$

Proof of Lemma 6.4. Notice upfront that $\bigcup_{i} B_{i} \neq[n]$. Otherwise there would exist $i_{1}, \ldots, i_{s}$ such that $i_{t} \in B_{i_{t+1}}, 1 \leq t \leq s\left(i_{s+1}=i_{1}\right)$, and - using repeatedly the property of the sets $B_{i}$ - we would get that, say, $i_{1} \in B_{i_{2}}$ and $i_{2} \in B_{i_{1}}$; hence $i_{2} \in B_{i_{2}}$, a contradiction.

Let $U_{1}, \ldots, U_{n}$ be independent $[0,1]$-uniform random variables. Let a random permutation $\omega$ be defined by

$$
\omega(i)=k \Longleftrightarrow U_{i} \text { is the } k^{\text {th }} \text { smallest amongst } U_{1}, \ldots, U_{n} .
$$

Clearly $\omega$ is distributed uniformly, and then so is $\pi:=\omega^{-1}$. With $\pi$ so defined, we obtain

$$
\begin{aligned}
\left\{E_{i}(\pi) \supseteq B_{i}, \forall i \leq n\right\} & =\left\{\pi^{-1}(i)>\pi^{-1}(j), \forall j \in B_{i}, i \leq n\right\} \\
& =\left\{U_{i}>U_{j}, \forall j \in B_{i}, i \leq n\right\} .
\end{aligned}
$$

Hence, the probability in question equals

$$
P\left(U_{i}>U_{j}, \forall j \in B_{i}, i \leq n\right) .
$$

We write this probability as the $n$-dimensional integral

$$
\begin{gathered}
P\left(U_{i}>U_{j}, \forall j \in B_{i}, i \leq n\right)=\int \underset{D}{\ldots} \int d x_{1} \cdots d x_{n}, \\
D=\left\{\left(x_{1}, \ldots, x_{n}\right) \in[0,1]^{n}: x_{i}>x_{j}, \forall j \in B_{i}, i \leq n\right\} .
\end{gathered}
$$

Since $\bigcup_{i} B_{i} \neq[n]$, we can choose an index $k \in[n]$ such that $k \notin B_{i}$ for all $i$. Then we may rewrite the integral above as

$$
\begin{gathered}
\int_{0}^{1}\left(\int_{D\left(x_{k}\right)} \cdots \int d x_{1} \cdots d x_{k-1} d x_{k+1} \cdots d x_{n}\right) d x_{k}, \\
D\left(x_{k}\right)=\left\{\left(x_{1}, \ldots, x_{k-1}, x_{k+1}, \ldots, x_{n}\right) \in[0,1]^{n-1}: x_{i}>x_{j}, \forall j \in B_{i}, i \leq n\right\} .
\end{gathered}
$$

On $D\left(x_{k}\right)$, the only inequalities involving $x_{k}$ are of the form $x_{k}>x_{j}, j \in B_{k}$. This suggests scaling those $x_{j}$ by $x_{k}$, i.e. introducing new variables $t_{j}:=x_{j} / x_{k}$, so that $t_{j} \in[0,1], j \in B_{k}$. To keep notation uniform, let us also replace the remaining $x_{i}, i \notin B_{k} \cup\{k\}$, with $t_{i}$. Let $\mathfrak{D}\left(x_{k}\right)$ denote the integration region for the new variables $t_{i}, i \neq k$. Explicitly, the constraints $x_{j}<x_{k}, j \in B_{k}$, become $t_{j}<1$, $j \in B_{k}$. Obviously each listed constraint $x_{a}<x_{b}\left(a, b \in B_{k}\right)$ is replaced, upon scaling, with $t_{a}<t_{b}$. We only rename the other variables, so every constraint $x_{a}<x_{b}\left(a, b \notin B_{k}\right)$ similarly becomes $t_{a}<t_{b}$. By the property of the sets $B_{i}$, there are no inequalities $x_{a}>x_{b}, a \in B_{k}, b \notin B_{k}$ (since the presence of this inequality implies $b \in B_{a}$ ). The only remaining inequalities are all of the type $x_{a}<x_{b}$, $a \in B_{k}, b \notin B_{k}$. In the new variables, such a constraint becomes $x_{k} t_{a}<t_{b}$, and it is certainly satified if $t_{a}<t_{b}$, as $x_{k} \leq 1$. Hence, $\mathfrak{D}\left(x_{k}\right) \supseteq D^{*}$, where

$$
D^{*}:=\left\{\left(t_{1}, \ldots, t_{k-1}, t_{k+1}, \ldots, t_{n}\right) \in[0,1]^{n-1}: t_{i}>t_{j}, \forall j \in B_{i}, i \neq k\right\},
$$


and $D^{*}$ does not depend on $x_{k}$ ! Observing that the constraints that determine $D^{*}$ are those for $D$ with the constraints $x_{i}<x_{k}, i \in B_{k}$, removed, we conclude that the innermost integral over $D\left(x_{k}\right)$ is bounded below by

$$
x_{k}^{\left|B_{k}\right|} P\left(U_{i}>U_{j}, \forall j \in B_{i}, i \neq k\right) .
$$

$\left(x_{k}^{\left|B_{k}\right|}\right.$ is the Jacobian of the linear transformation $\left\{x_{i}\right\}_{i \neq k} \rightarrow\left\{t_{i}\right\}_{i \neq k}$.) Integrating with respect to $x_{k}$, we arrive at

$$
P\left(U_{i}>U_{j}, \forall j \in B_{i}, i \leq n\right) \geq \frac{1}{\left|B_{k}\right|+1} \cdot P\left(U_{i}>U_{j}, \forall j \in B_{i}, i \neq k\right) .
$$

By induction on the number of sets $B_{i}$, with Lemma 6.1 providing the basis of the induction and (6.1) providing the inductive step, we get

$$
P\left(U_{i}>U_{j}, \forall j \in B_{i}, i \leq n\right) \geq \prod_{i=1}^{n} \frac{1}{\left|B_{i}\right|+1} .
$$

The rest is short. First, by Lemma 6.4,

$$
\begin{aligned}
P\left(E_{i}(\pi) \supseteq E_{i}(\sigma), \forall i \leq n\right) & =E\left[\left.P\left(E_{i}(\pi) \supseteq B_{i}, \forall i \leq n\right)\right|_{B_{i}=E_{i}(\sigma)}\right] \\
& \geq E\left[\prod_{i=1}^{n} \frac{1}{\left|E_{i}(\sigma)\right|+1}\right] .
\end{aligned}
$$

Since the cardinalities $\left|E_{i}(\sigma)\right|$ are independent, the last expected value equals

$$
\prod_{i=1}^{n} E\left[\frac{1}{\left|E_{i}(\sigma)\right|+1}\right]=\prod_{i=1}^{n}\left(\frac{1}{i} \sum_{j=0}^{i-1} \frac{1}{j+1}\right)=\prod_{i=1}^{n} \frac{H(i)}{i} ;
$$

for the second to last equality, see the proof of Lemma 6.2.

Note. Let $\mathcal{P}$ be a poset on $[n]$, and put $B_{i}:=\{j \in \mathcal{P}: j<i$ in $\mathcal{P}\} . B_{i} \cup\{i\}$ is called the order ideal at $i$. By the properties of $\mathcal{P}$, the $B_{i}$ 's satisfy the hypotheses of Lemma 6.4, so letting $e(\mathcal{P})$ denote the number of linear extensions of $\mathcal{P}$ we get

$$
\begin{aligned}
P\left(E_{i}(\pi) \supseteq B_{i}, \forall i \leq n\right) & =\frac{\left|\left\{\omega: \omega(i)>\omega(j), \forall j \in B_{i}, i \leq n\right\}\right|}{n !} \\
& =\frac{e(\mathcal{P})}{n !} \geq \prod_{i=1}^{n} \frac{1}{\left|B_{i}\right|+1} .
\end{aligned}
$$

Thus we have proved

Corollary 2. For a poset $\mathcal{P}$ with $n$ elements,

$$
e(\mathcal{P}) \geq n ! / \prod_{i=1}^{n} d(i), \quad d(i):=\mid\{j \in \mathcal{P}: j \leq i \text { in } \mathcal{P}\} \mid .
$$

In a very special case of $\mathcal{P}$, whose Hasse diagram is a forest of rooted trees with edges directed away from the roots, this simple bound is actually the value of $e(\mathcal{P})$ ([24, p. 312, ex. 1], [18, sect. 5.1.4, ex. 20], 5]). There exist better bounds for the number of linear extensions in the case of the Boolean lattice (see Brightwell and Tetali [9], Kleitman and Sha [17]), but nothing has been done in the way of 
improving this bound for $\mathcal{P}=\mathcal{P}(\sigma)$, the permutation-induced poset. Indeed, our proof of the lower bound for $P_{n}^{*}$ used only the universal bound of Corollary 2 , and not one specific to this special poset. So this begs the question of whether we might improve the bound in this case, and consequently improve on our lower estimate for $P_{n}^{*}$. We are presently working in this direction.

\section{NumERICS}

From computer-generated data we have collected, it appears that our $O\left(n^{-2}\right)$ upper bound correctly predicts the qualitative behavior of $P(\pi \leq \sigma)$. The data suggests that $P(\pi \leq \sigma)$ is of exact order $n^{-(2+\delta)}$ for some $\delta \in[0.5,1]$, which begs the question of how to improve on our current bound. Writing $P_{n}=P(\pi \leq \sigma)$, below is a graph (based on this numerical experimentation) exhibiting convergence to the exponent $-a$ in the asymptotic equation $P_{n} \sim c n^{-a}, c>0$ a constant, and

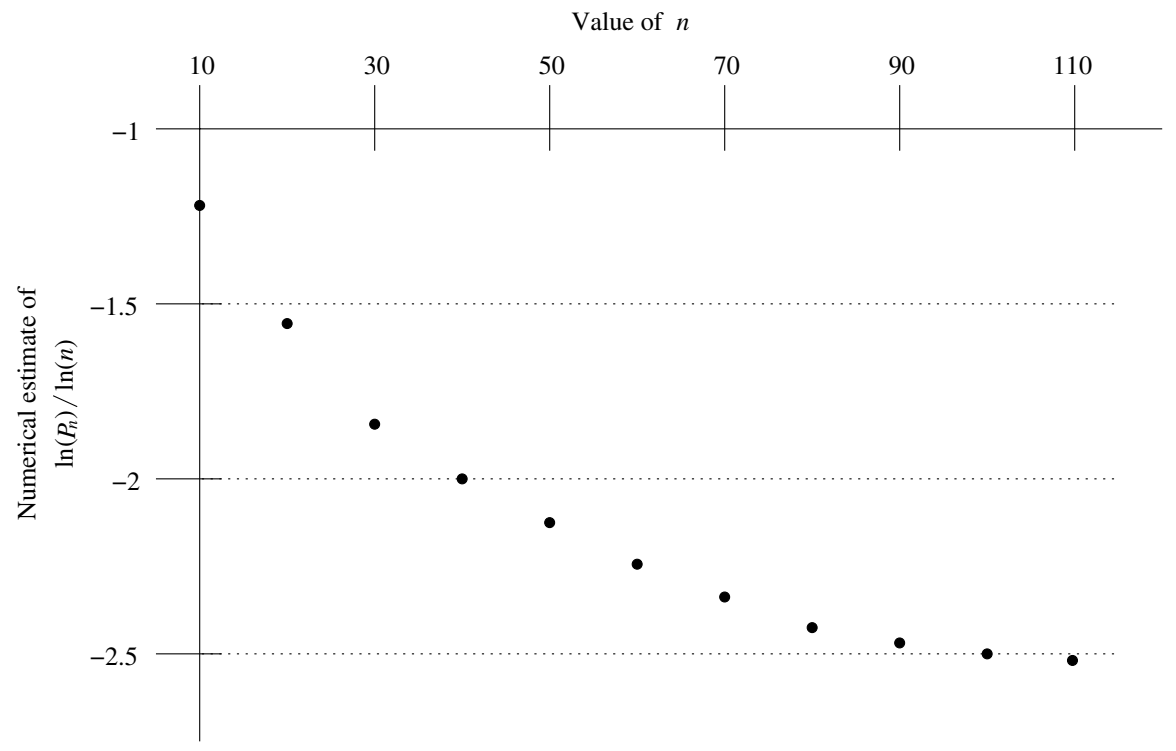

$-a$ appears to be near -2.5 . Here is a portion of the accompanying table used to generate this graph:

\begin{tabular}{r|r|l|l}
\multicolumn{1}{c|}{$n$} & \multicolumn{1}{|c|}{$R_{n}$} & Estimate of $P_{n} \approx \frac{R_{n}}{10^{9}}$ & Estimate of $\ln \left(P_{n}\right) / \ln n$ \\
\hline 10 & 61589126 & $0.0615891 \ldots$ & $-1.21049 \ldots$ \\
30 & 1892634 & $0.0018926 \ldots$ & $-1.84340 \ldots$ \\
50 & 233915 & $0.0002339 \ldots$ & $-2.13714 \ldots$ \\
70 & 50468 & $0.0000504 \ldots$ & $-2.32886 \ldots$ \\
90 & 14686 & $0.0000146 \ldots$ & $-2.47313 \ldots$ \\
110 & 5174 & $0.0000051 \ldots$ & $-2.58949 \ldots$
\end{tabular}

Here, $R_{n}$ is the number of pairs $(\pi, \sigma)$ out of $10^{9}$ randomly-generated pairs such that we had $\pi \leq \sigma$. We have also utilized the computer to find the actual probability $P_{n}$ for $n=1,2, \ldots, 9$. Below is a table of these true proportions. 


\begin{tabular}{r|r|c}
$n$ & $(n !)^{2} P_{n}$ & $P_{n}$ \\
\hline 1 & 1 & $1.00000 \ldots$ \\
2 & 3 & $0.75000 \ldots$ \\
3 & 19 & $0.52777 \ldots$ \\
4 & 213 & $0.36979 \ldots$ \\
5 & 3781 & $0.26256 \ldots$ \\
6 & 98407 & $0.18982 \ldots$ \\
7 & 3550919 & $0.13979 \ldots$ \\
8 & 170288585 & $0.10474 \ldots$ \\
9 & 10501351657 & $0.07974 \ldots$
\end{tabular}

Concerning the weak order, computer-generated data suggests that $P(\pi \preceq \sigma)$ is of exact order $(0.3)^{n}$. So our current upper bound $O\left((0.362)^{n}\right)$ is a qualitative match for $P(\pi \preceq \sigma)$, but it appears that improvements are possible here also. Writing $P_{n}^{*}=P(\pi \preceq \sigma)$, below is a graph (based on our numerical experiments) exhibiting convergence to the ratio $\rho$ in the asymptotic equation $P_{n}^{*} \sim c \rho^{n}, c>0$ a constant,

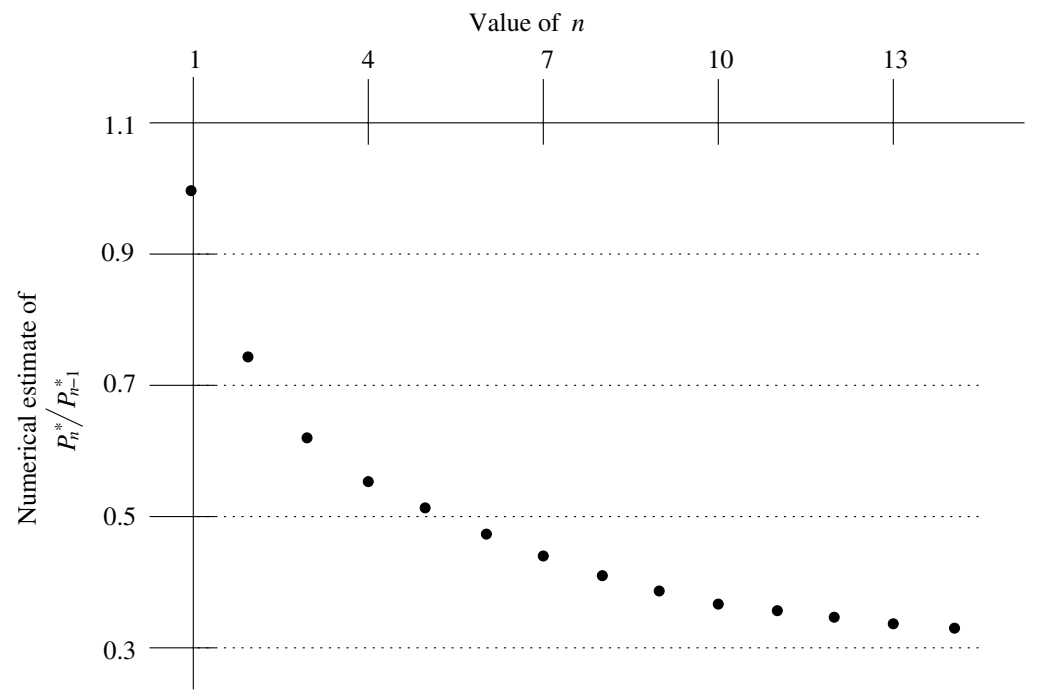

and $\rho$ appears to be near 0.3 . Here is a portion of the accompanying table used to generate this graph:

\begin{tabular}{r|r|l|l}
$n$ & \multicolumn{1}{|c|}{$R_{n}^{*}$} & Estimate of $P_{n}^{*} \approx \frac{R_{n}^{*}}{10^{9}}$ & Estimate of $P_{n}^{*} / P_{n-1}^{*}$ \\
\hline 10 & 1538639 & $0.0015386 \ldots$ & $0.368718 \ldots$ \\
11 & 541488 & $0.0005414 \ldots$ & $0.351926 \ldots$ \\
12 & 184273 & $0.0001842 \ldots$ & $0.340308 \ldots$ \\
13 & 59917 & $0.0000599 \ldots$ & $0.325153 \ldots$ \\
14 & 18721 & $0.0000187 \ldots$ & $0.312448 \ldots$ \\
15 & 5714 & $0.0000057 \ldots$ & $0.305218 \ldots$ \\
16 & 1724 & $0.0000017 \ldots$ & $0.301715 \ldots$
\end{tabular}

Here, $R_{n}^{*}$ is defined analogously to $R_{n}$ above. Below is a table with the true proportion $P_{n}^{*}$ for $n=1,2, \ldots, 9$. 


\begin{tabular}{r|r|l}
$n$ & $(n !)^{2} P_{n}^{*}$ & $P_{n}^{*}$ \\
\hline 1 & 1 & $1.00000 \ldots$ \\
2 & 3 & $0.75000 \ldots$ \\
3 & 17 & $0.47222 \ldots$ \\
4 & 151 & $0.26215 \ldots$ \\
5 & 1899 & $0.13187 \ldots$ \\
6 & 31711 & $0.06117 \ldots$ \\
7 & 672697 & $0.02648 \ldots$ \\
8 & 17551323 & $0.01079 \ldots$ \\
9 & 549500451 & $0.00417 \ldots$
\end{tabular}

Surprisingly, our Theorem 1.2 lower bound for $P_{n}^{*}$ is quite good for these smallish values of $n$ :

\begin{tabular}{r|r|l}
$n$ & $(n !)^{2} \prod_{i=1}^{n}(H(i) / i)$ & $\prod_{i=1}^{n}(H(i) / i)$ \\
\hline 1 & $1.0 \ldots$ & $1.00000 \ldots$ \\
2 & $3.0 \ldots$ & $0.75000 \ldots$ \\
3 & $16.5 \ldots$ & $0.45833 \ldots$ \\
4 & $137.5 \ldots$ & $0.23871 \ldots$ \\
5 & $1569.8 \ldots$ & $0.10901 \ldots$ \\
6 & $23075.9 \ldots$ & $0.04451 \ldots$ \\
7 & $418828.3 \ldots$ & $0.01648 \ldots$ \\
8 & $9106523.1 \ldots$ & $0.00560 \ldots$ \\
9 & $231858583.9 \ldots$ & $0.00176 \ldots$
\end{tabular}

\section{ACKNOWLEDGMENTS}

This work was inspired by a thought-provoking talk Mark Skandera gave at the MIT Combinatorics Conference honoring Richard Stanley's $60^{\text {th }}$ birthday. We are grateful to Mark for an enlightening follow-up discussion of comparability criteria for the Bruhat order. We thank Sergey Fomin for encouragement and for introducing us to an instrumental notion of the permutation-induced poset, and also for many insightful suggestions regarding the history of the Bruhat order. We gladly accepted Sergey's suggestion to modify the initial title as to more accurately reflect the paper's contents. Without Ed Overman's invaluable guidance we would not have been able to obtain the numerical results in this paper. Craig Lennon gave us an idea for proving an exponential lower bound in the case of the Bruhat order. We thank Miklós Bóna for his interest in the paper and for drawing our attention to the lower bound for the number of linear extensions in Richard Stanley's book.

\section{REFERENCES}

1. C. Berge, Principles of Combinatorics, Vol. 72 of Mathematics in Science and Engineering: A Series of Monographs and Textbooks, Academic Press, Inc., New York, 1971. MR0270922 (42:5805)

2. P. Billingsley, Probability and Measure, $3^{\text {rd }}$ ed., John Wiley \& Sons, Inc., New York, 1995. MR.1324786 (95k:60001)

3. A. Buörner, F. Brenti, Combinatorics of Coxeter Groups, Springer, New York, 2005. MR2133266 (2006d:05001)

4. A. Björner, F. Brenti, An Improved Tableau Criterion for Bruhat Order, Electron. J. Combin. 3 (1996), \#R22, 5 pp. (electronic). MR.1399399 (97c:05160)

5. A. BJÖRner, M. WACHS, q-Hook Length Formulas for Forests, Journal of Combinatorial Theory, Series A 52 (1989), pp. 165-187. MR1022316 (91e:05013) 
6. A. BJöRner, Orderings of Coxeter Groups, in Combinatorics and Algebra (C. GreEne, ed.), Vol. 34 of Contemp. Math., American Mathematical Society, Providence, RI, 1984, pp. 175195. MR777701 (86i:05024)

7. M. BónA, Personal communication about an early preprint, August 2006.

8. M. BónA, Combinatorics of Permutations, Discrete Mathematics and its Applications, Chapman \& Hall/CRC, Boca Raton, 2004. MR2078910 (2005f:05001)

9. G. Brightwell, P. Tetali, The Number of Linear Extensions of the Boolean Lattice, Order 20 (2003), pp. 333-345. MR2079149 (2005d:06002)

10. V. Deodhar, Some Characterizations of Bruhat Ordering on a Coxeter Group and Determination of the Relative Möbius Function, Inventiones Math. 39 (1977), pp. 187-198. MR0435249 (55:8209)

11. B. Drake, S. Gerrish, M. Skandera, Two New Criteria for Comparison in the Bruhat Order, Electron. J. Combin. 11 (2004), \#N6, 4 pp. (electronic). MR2056088

12. C. Ehresmann, Sur la topologie de certains espaces homogènes, Ann. Math. 35 (1934), pp. 396-443. MR.1503170

13. S. Fomin, Personal communication at the Michigan University Combinatorics Seminar, April 2005.

14. W. Fulton, Young Tableaux: With Applications to Representation Theory and Geometry, Vol. 35 of London Mathematical Society Student Texts, Cambridge University Press, New York, 1997. MR1464693 (99f:05119)

15. J. E. Humphreys, Reflection Groups and Coxeter Groups, Cambridge Studies in Advanced Mathematics, No. 29, Cambridge University Press, Cambridge, 1990. MR 1066460 (92h:20002)

16. S. Janson, T. Łuczak, A. Ruciński, Random Graphs, John Wiley \& Sons, Inc., New York, 2000. MR 1782847 (2001k:05180)

17. D. Kleitman, J. Sha, The Number of Linear Extensions of Subset Ordering, Discrete Math. 63 (1987), pp. 271-279. MR885505 (88c:05016)

18. D. Knuth, Sorting and Searching: The Art of Computer Programming, Vol. III, AddisonWesley Publishing Company, Inc., Reading, MA, 1973. MR0445948 (56:4281)

19. A. Lascoux, M. P. Schützenberger, Treillis et bases des groupes de Coxeter, Electron. J. Combin. 3 (1996), \#R27, 35 pp. (electronic). MR.1395667(98c:05168)

20. V. G. Mikhailov, V. A. Vatutin, Limit Theorems for the Number of Empty Cells in an Equiprobable Scheme for Group Allocation of Particles, Teor. Veroyatnost. i Primenen. 27 (1982), pp. 684-692 (Russian); Theor. Probab. Appl. 27, pp. 734-743 (English transl.). MR.681461 (84c:60020)

21. B. Pittel, Confirming Two Conjectures About the Integer Partitions, Journal of Combinatorial Theory, Series A 88 (1999), pp. 123-135. MR.1713480 (2000k:11114)

22. B. Pittel, Random Set Partitions: Asymptotics of Subset Counts, Journal of Combinatorial Theory, Series A 79 (1997), pp. 326-359. MR1462562 (98h:05021)

23. G. Pólya, G. Szegö, Problems and Theorems in Analysis, Springer, New York, 1976.

24. R. Stanley, Enumerative Combinatorics, Vol. I, Cambridge University Press, Cambridge, 1997. MR 1442260 (98a:05001)

25. L. TAKacs, Combinatorial Methods in the Theory of Stochastic Processes, John Wiley \& Sons, Inc., New York, 1967. MR0217858(36:947)

Department of Mathematics, The Ohio State University, 231 W. 18th Avenue, ColumBUs, OHIO 43210

E-mail address: hammett@math.ohio-state.edu

Department of Mathematics, The Ohio State University, 231 W. 18th Avenue, ColumBUS, Оніо 43210

E-mail address: bgp@math.ohio-state.edu 